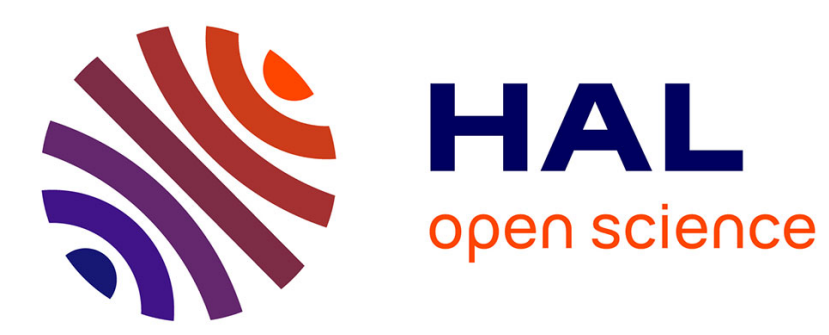

\title{
Modal basis approaches in shape and topology optimization of frequency response problems
}

Grégoire Allaire, Georgios Michailidis

\section{To cite this version:}

Grégoire Allaire, Georgios Michailidis. Modal basis approaches in shape and topology optimization of frequency response problems. International Journal for Numerical Methods in Engineering, 2018, 113 (8), pp.1258-1299. hal-01354162

\section{HAL Id: hal-01354162 \\ https://hal.science/hal-01354162}

Submitted on 17 Aug 2016

HAL is a multi-disciplinary open access archive for the deposit and dissemination of scientific research documents, whether they are published or not. The documents may come from teaching and research institutions in France or abroad, or from public or private research centers.
L'archive ouverte pluridisciplinaire HAL, est destinée au dépôt et à la diffusion de documents scientifiques de niveau recherche, publiés ou non, émanant des établissements d'enseignement et de recherche français ou étrangers, des laboratoires publics ou privés. 


\title{
Modal basis approaches in shape and topology optimization of frequency response problems
}

\author{
Grégoire Allaire \\ CMAP, Ecole Polytechnique, CNRS, Université Paris-Saclay, \\ 91128 Palaiseau, France \\ (gregoire.allaire@polytechnique.fr) \\ Georgios Michailidis \\ SIMaP-Université de Grenoble, INPG, \\ 38000 Grenoble, France \\ (georgios.michailidis@simap.grenoble-inp.fr)
}

July 14, 2016

\begin{abstract}
The optimal design of mechanical structures subject to periodic excitations within a large frequency interval is quite challenging. In order to avoid bad performances for non-discretized frequencies, it is necessary to finely discretize the frequency interval, leading to a very large number of state equations. Then, if a standard adjoint-based approach is used for optimization, the computational cost (both in terms of CPU and memory storage) may be prohibitive for large problems, especially in three space dimensions. The goal of the present work is to introduce two new non-adjoint approaches for dealing with frequency response problems in shape and topology optimization. In both cases, we rely on a classical modal basis approach to compute the states, solutions of the direct problems. In the first method, we do not use any adjoint but rather directly compute the shape derivatives of the eigenmodes in the modal basis. In the second method, we compute the adjoints of the standard approach by using again the modal basis. The numerical cost of these two new strategies are much smaller than the usual ones if the number of modes in the modal basis is much smaller than the number of discretized excitation frequencies. We present numerical examples for the minimization of the dynamic compliance in two and three space dimensions.
\end{abstract}

Keywords: shape and topology optimization, frequency response, level-set method

\section{Introduction}

Shape and topology optimization techniques $[1,5,9]$ find today extensive applications in industry related to product design. Its incorporation in the design cycle can significantly reduce the required time for the conception of a mechanical part and improve its performance. Especially when the mechanical framework of the application is complicated enough, relying only on the experience and intuition of engineers frequently proves to be not efficient enough. In such cases, performancedriven automated design techniques can provide a powerful remedy, allowing designers to enrich their knowledge and result in better designs.

Frequency response problems appear in a wide variety of industries, such as the automotive and the aeronautic sectors. Such problems are characterized by a Helmholtz state equation, featuring 
the excitation frequency and possibly some damping, and furthermore by the fact that usually one is not interested by a single frequency but rather by a whole interval of excitation frequencies. Discretization of the frequency range yields a large number of Helmholtz state equations. Contrary to multiple loads optimization where the state equation is the same and only the right hand sides vary, frequency response optimization leads to a collection of different partial differential equations or rigidity matrices. As a consequence, the computational cost of frequency response optimization is very large and even prohibitive for industrial applications with large-scale problems. This is all the more an issue since the frequency interval must be finely discretized otherwise intermediate frequencies in a gap between two discretized frequencies may lead to completely suboptimal performances.

In order to reduce the computational cost of frequency response optimization, a classical approach is to work with a modal basis. In other words, for a given structure, one computes its first eigenfrequencies and eigenmodes (without damping) in a range covering the entire excitation frequency interval. Then, the Helmholtz state equations (with damping) are solved by a Galerkin method with this modal basis. In the context of shape and topology optimization, this additionally requires the solution of an adjoint problem for every eigenfrequency. Since the number of eigenfrequencies of the modal basis can be quite large, the total cost of this approach can be indeed prohibitive for industrial applications.

There is already a vast literature for topology optimization of frequency response problems and we give a brief and non exhaustive account of it. Early works were done in the framework of the homogenization method: Ma et al. [19], [20] minimized the mean compliance in a frequency interval using a direct and a modal analysis, while Min et al. [21] used an optimality criteria method to minimize the dynamic compliance, working in the time domain. In the context of plate thickness optimization, Jog [16] worked on the dynamic compliance and the frequency amplitude. Later, many more works appeared using the SIMP method. Tcherniak [32] presented a method, based on modal analysis, for the design of resonating structures. Olhoff et al. [23] and Yoon [34] studied the dynamic compliance minimization. A very interesting paper was written by Jensen [15] in order to accelerate the sensitivity computations: he proposed to use Padè approximants for the solution of dynamic problems, avoiding the costly calculation of adjoint states for many excitation frequencies. Finally, in the level-set framework (that we adopt in the present article) the first work about frequency response problems is that of Shu et al. [29].

Here, our main goal is to present two new methods for the treatment of frequency response problems, which do not require the computation of adjoint states, and thus accelerate significantly the optimization process and make feasible the application of shape and topology optimization on industrial frequency response problems. Although the proposed methods can be applied in the framework of density-based methods too, we use here the level-set method for the shape description $[2,3,25,28,33]$, in order to benefit both from its geometric advantage of a clear definition of a shape and to avoid possible ghost modes in the modal analysis, localized in region of intermediate densities.

The content of the present paper is as follows. Section 2 is devoted to a general presentation of the mechanical framework and of the optimization setting for frequency response problems. Section 3 is a brief introduction to the basic ingredients for shape and topology optimization via the level-set method. For reasons of completeness, the standard adjoint approach is recalled in Section 4. The main conclusion is that it leads to as many adjoint states as there are discrete excitation frequencies, which is totally prohibitive from a numerical point of view. Section 5 describes what is more or less the state of the art in frequency response problems. A modal basis approach is used to compute the solutions of the Helmholtz state equations. By considering the spectral equations as the effective state equations, the optimization process leads to consider new adjoint equations, the number of which is roughly the number of eigenfrequencies in the modal basis. Although it is a serious improvement with respect to the previous standard approach of 
Section 4, it is still a very costly strategy in terms of computation time and memory storage. Our first new self-adjoint method is presented in Section 6: it is based on the approximation of the eigenmodes' shape derivatives in the modal basis and requires no adjoint equations. Note that this method, as well as the next one, can cope with damping as long as the damping operator is diagonal in the modal basis, as is often the case in numerical practice. A second new self-adjoint method is proposed in Section 7. Its principle is quite simple: it starts from the standard shape derivative obtained in Section 4 and replace the direct and adjoint states by their approximation in the modal basis, therefore eliminating the need of solving any adjoint equations. A comparison of all these approaches is performed in Section 8 in terms of complexity or operation counts. It shows that our two proposed self-adjoint methods outperform the other approaches as soon as the number of discretized excitation frequencies and the number of eigenfrequencies in the modal basis are large. Of course, a key issue for numerical efficiency is to have access to a fast and accurate algorithm to compute the modal basis. Section 9 is an assessment of the modal basis approach. We discuss convergence issues in terms of the number of eigenmodes and of the discretization step for the excitation frequency interval. Finally, numerical results in two and three space dimensions are shown in Section 10, which validates our proposed approach. We focus on dynamic compliance minimization problems but other objective functions would work as well in our setting. We also check that multiple eigenvalues are not an issue from a numerical point of view, although it is known to be a delicate point when it comes to their differentiability properties.

\section{Setting of the problem}

\subsection{State equation}

Consider a structure, occupying a bounded domain $\Omega \subset \mathbb{R}^{d}$, with $d=2,3$, which vibrates under the application of a periodic time-harmonic load $f(x, t)$ at some part of its boundary $\Gamma_{N} \subset \partial \Omega$. The structure is fixed at $\Gamma_{D} \subset \partial \Omega$, while the rest of its boundary, denoted $\Gamma$, is free and subject to optimization. The corresponding displacement field $u(x, t)$ is a solution of the system

$$
\left\{\begin{aligned}
\rho(x) \ddot{u}+c(x) \dot{u}-\operatorname{div}(A e(u)) & =0 & & \text { in } \Omega \times \mathbb{R}^{+}, \\
u & =0 & & \text { on } \Gamma_{D} \times \mathbb{R}^{+}, \\
(A e(u)) n & =f(t) & & \text { on } \Gamma_{N} \times \mathbb{R}^{+}, \\
(A e(u)) n & =0 & & \text { on } \Gamma \times \mathbb{R}^{+},
\end{aligned}\right.
$$

where $\rho(x)>0$ is the material density, $c(x) \geq 0$ the damping function, $A$ the isotropic Hooke's law, $e(u)=\left(\nabla u+(\nabla u)^{T}\right) / 2$ the strain tensor and the $\operatorname{dot}\left({ }^{\circ}\right)$ denotes derivation with respect to time. Since we are looking for a time-harmonic displacement $u(x, t)$, we did not include initial conditions in (1). To facilitate the analysis, we work with complex-valued functions (with $i=\sqrt{-1}$ ). Assume the complex loading to be of the type

$$
f(x, t)=F(x) e^{i \omega t}, \quad F=f_{\operatorname{Re}}+i f_{\operatorname{Im}}, f_{\operatorname{Re}}, f_{\operatorname{Im}} \in \mathbb{R}
$$

where $\omega>0$ is the excitation frequency and $F$ the complex loading amplitude. Similarly we look for a complex time-harmonic solution of (1) which reads as

$$
u(x, t)=U(x) e^{i \omega t}, \quad U=u_{\mathrm{Re}}+i u_{\mathrm{Im}}, u_{\mathrm{Re}}, u_{\mathrm{Im}} \in \mathbb{R} .
$$


One recovers a real-valued solution of (1) by taking the real part $\operatorname{Re}(u)=u_{\operatorname{Re}} \cos (\omega t)-u_{\operatorname{Im}} \sin (\omega t)$. Substituting (3) in (1), we obtain the equation satisfied by the displacement amplitude $U$ :

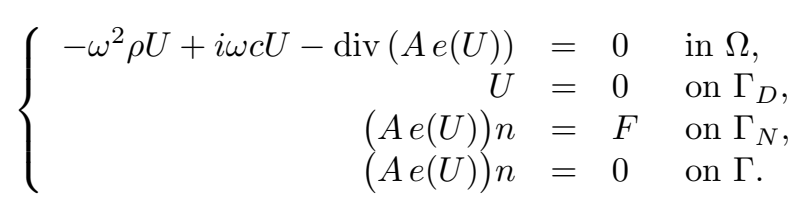

In the sequel we shall always work in the frequency domain, rather than in the time domain. Namely, we use (4) as the state equation, instead of (1). Separating the real and imaginary parts, we get the following two coupled systems of PDE's:

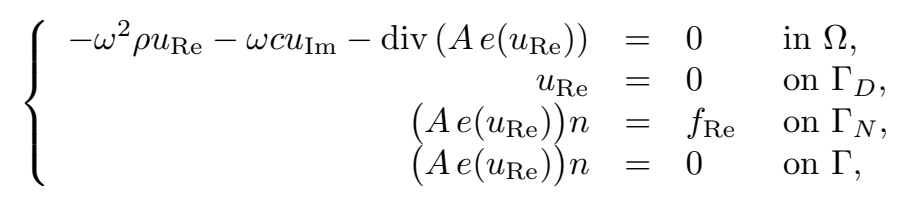

and

$$
\left\{\begin{aligned}
-\omega^{2} \rho u_{\operatorname{Im}}+\omega c u_{\mathrm{Re}}-\operatorname{div}\left(A e\left(u_{\operatorname{Im}}\right)\right) & =0 & & \text { in } \Omega, \\
u_{\operatorname{Im}} & =0 & & \text { on } \Gamma_{D}, \\
\left(A e\left(u_{\operatorname{Im}}\right)\right) n & =f_{\operatorname{Im}} & & \text { on } \Gamma_{N}, \\
\left(A e\left(u_{\operatorname{Im}}\right)\right) n & =0 & & \text { on } \Gamma .
\end{aligned}\right.
$$

Remark 2.1. For given loads $f_{\operatorname{Re}}, f_{\operatorname{Im}} \in L^{2}\left(\Gamma_{N}\right)^{d}$ and in the presence of damping, i.e. $c(x) \geq 0$ and $c \neq 0$, the system of equations (5), (6) has a unique solution $u_{\mathrm{Re}}, u_{\mathrm{Im}} \in H^{1}(\Omega)^{d}$ (see e.g. Lemma 2.6.6 in [22]). In the undamped case $(c=0)$, a unique solution exists when the frequency of the external loading does not coincide with an eigenfrequency of the structure. Note that in the sequel, we shall often replace the damping multiplicative coefficient $c(x)$ by a diagonal operator $C$ in the modal basis (see Section 5 for more details).

Remark 2.2. For simplicity, and because it is enough in many applications, we consider only surface loads on $\Gamma_{N}$. However, it is not a restriction and all our analysis in the present paper can be extended mutatis mutandis to the case of bulk loads in a non-optimizable part of the domain $\Omega$ (the case of loads touching the optimizable free boundary is slightly different, although amenable to our approach).

Remark 2.3. After discretization, for example using the finite element method, equations (5) and (6) become

$$
\left[\begin{array}{cc}
K-\omega^{2} M & -\omega C \\
\omega C & K-\omega^{2} M
\end{array}\right]\left[\begin{array}{l}
u_{\operatorname{Re}} \\
u_{\operatorname{Im}}
\end{array}\right]=\left[\begin{array}{c}
f_{\operatorname{Re}} \\
f_{\operatorname{Im}}
\end{array}\right],
$$

which, with obvious notations, can also be written in the form

$$
S(\omega) U=F \quad \text { with } \quad S(\omega)=K+i \omega C-\omega^{2} M,
$$

where the complex matrix $S(\omega)$ is called the dynamic stiffness matrix. The matrices $K, C, M$ are real symmetric and $K, M$ are positive definite. Very often, at the discrete level, the matrix $C$ is replaced by a linear combination of $K$ and $M$.

\subsection{Optimization problem}

Our goal is to find a shape $\Omega$, belonging to an admissible set $\mathcal{U}_{a d}$ that minimizes an objective function $J(\Omega)$, which depends on the shape through the solution $\left(u_{\operatorname{Re}}, u_{\mathrm{Im}}\right)$ of equation (4). The shape optimization problem reads

$$
\min _{\Omega \in \mathcal{U}_{a d}} J(\Omega)
$$


Very often, we shall abuse notations and write

$$
J(\Omega)=J\left(u_{\mathrm{Re}}(\Omega), u_{\operatorname{Im}}(\Omega)\right) .
$$

A famous example of such a functional, for frequency response applications, is the so-called dynamic compliance, which reads

$$
J(\Omega)=\int_{\omega_{\min }}^{\omega_{\max }} \int_{\Gamma_{N}}\left(f_{\operatorname{Im}} \cdot u_{\operatorname{Re}}(\omega)-f_{\operatorname{Re}} \cdot u_{\operatorname{Im}}(\omega)\right) d s d \omega
$$

where $\left[\omega_{\min }, \omega_{\max }\right]$ denotes the interval of excitation frequencies, $0<\omega_{\min }<\omega_{\max }$. More general objective functions can be considered as well: our entire approach in the sequel can easily be extended. Their distinctive feature is that they depend on a full range of frequencies and not just a single one. In numerical practice, this frequency range will be discretized and the $\omega$-integral in (10) will be replaced by a sum over finitely many excitation frequencies $\tilde{\omega}_{i}, 1 \leq i \leq N_{\omega}$. The number of discrete frequencies $N_{\omega}$ is usually very large (typically of the order of 100 to 1000 , corresponding to a frequency step of one Hertz), which makes the evaluation of the objective function quite expensive since it requires to solve $N_{\omega}$ state equations of the type (4).

Remark 2.4. For the sake of completeness, we give here a brief physical interpretation of the dynamic compliance for a specific excitation frequency $\omega$. Defining the instantaneous input power due to the loading as

$$
P_{\text {inp }}=\int_{\Gamma_{N}}(\dot{u} \cdot f) d s=\int_{\Gamma_{N}}-\omega\left(u_{\mathrm{Re}} \sin (\omega t)+u_{\mathrm{Im}} \cos (\omega t)\right) \cdot\left(f_{\mathrm{Re}} \cos (\omega t)-f_{\operatorname{Im}} \sin (\omega t)\right) d s,
$$

the energy change over one excitation cycle due to the loading reads

$$
\Delta E_{i n p}=\int_{0}^{\frac{2 \pi}{\omega}} P_{i n p} d t=\pi \int_{\Gamma_{N}}\left(u_{\mathrm{Re}} \cdot f_{\mathrm{Im}}-u_{\mathrm{Im}} \cdot f_{\mathrm{Re}}\right) d s .
$$

Omitting the factor $\pi$, the dynamic compliance is thus defined as

$$
C_{d y n}=\int_{\Gamma_{N}}\left(u_{\mathrm{Re}} \cdot f_{\mathrm{Im}}-u_{\mathrm{Im}} \cdot f_{\mathrm{Re}}\right) d s .
$$

\section{Shape and topology optimization framework}

In general, a shape and topology optimization method is characterized by two major ingredients: a method to describe the shape and a method to update it, optimizing its performance with respect to some pre-defined criteria. In this work, following the lead of $[3,33]$ we use the level-set method for the shape description and the Hadamard method of shape differentiation in order to deduce a descent direction, briefly described in the rest of this section.

\section{$3.1 \quad$ Level-set method}

The level-set method, developped by Osher and Sethian [26], uses an implicit representation of an evolving front as the zero level-set of an auxiliary function $\phi$. More precisely, assuming that the domain $\Omega$ of interest is a subset of a large working domain $D$, the level-set representation of $\Omega$ can be defined as

$$
\left\{\begin{aligned}
\phi(x)=0 & \Leftrightarrow x \in \partial \Omega \cap D, \\
\phi(x)<0 & \Leftrightarrow x \in \Omega, \\
\phi(x)>0 & \Leftrightarrow x \in(D \backslash \bar{\Omega}) .
\end{aligned}\right.
$$


The advection of the front (or shape boundary) with a normal velocity $V(x, t)$ is described in the level-set framework by the well-known Hamilton-Jacobi transport equation:

$$
\frac{\partial \phi}{\partial t}+V(x, t)|\nabla \phi|=0
$$

using an explicit second order upwind scheme $[24,27]$. In our optimization setting the time $t \in \mathbb{R}^{+}$ can be interpreted, after discretization, as a descent step.

\subsection{Shape sensitivity approach}

In shape and topology optimization, the normal velocity $V(x, t)$ used for the shape evolution in (12) is chosen so that the objective function decreases during the (pseudo-)time evolution. In a gradient flow approach, or gradient-based optimization method, one first needs to compute a shape derivative of the objective function by using the classical Hadamard method [1], [14], [30], [31].

Starting from a smooth reference open set $\Omega$, we consider variations of the type

$$
\Omega_{\theta}=(I d+\theta) \Omega
$$

with $\theta \in W^{1, \infty}\left(\mathbb{R}^{d}, \mathbb{R}^{d}\right)$.

Definition 3.1. The shape derivative of $J(\Omega)$ at $\Omega$ is defined as the Fréchet derivative in $W^{1, \infty}\left(\mathbb{R}^{d}, \mathbb{R}^{d}\right)$ at 0 of the application $\theta \rightarrow J((I d+\theta) \Omega)$, i.e.

$$
J((I d+\theta) \Omega)=J(\Omega)+J^{\prime}(\Omega)(\theta)+o(\theta) \quad \text { with } \quad \lim _{\theta \rightarrow 0} \frac{|o(\theta)|}{\|\theta\|}=0,
$$

where $\theta \rightarrow J^{\prime}(\Omega)(\theta)$ is a continuous linear form on $W^{1, \infty}\left(\mathbb{R}^{d}, \mathbb{R}^{d}\right)$.

A classical result (Hadamard's structure theorem) states that the shape derivative $J^{\prime}(\Omega)(\theta)$ depends only on the normal trace $\theta \cdot n$ on the boundary $\partial \Omega$. In fact, for a great variety of functionals, the shape derivative can be written in the form

$$
J^{\prime}(\Omega)(\theta)=\int_{\partial \Omega} \theta(s) \cdot n(s) V(s) d s
$$

where the integrand $V$ depends on the specific objective function and boundary conditions. Then, a descent direction can be found by advecting the shape in the direction $\theta(s)=-t V(s) n(s)$ for a small enough descent step $t>0$. For the new shape $\Omega_{t}=(\operatorname{Id}+t \theta) \Omega$, if $V \neq 0$, we can formally write

$$
J\left(\Omega_{t}\right)=J(\Omega)-t \int_{\partial \Omega} V(s)^{2} d s+\mathcal{O}\left(t^{2}\right)<J(\Omega)
$$

which guarantees a descent direction for small positive $t$.

Assumption. In the sequel, we assume that only the free boundary $\Gamma$ is allowed to be optimized and that the Dirichlet and Neumann parts of the boundary $\Gamma_{D}$ and $\Gamma_{N}$ are kept fixed. In other words, we assume that all vector fields $\theta$ satisfy $\theta=0$ on $\Gamma_{D}$ and $\Gamma_{N}$.

\section{Standard adjoint approach}

In this Section, we obtain the shape derivative of the dynamic compliance (10) by following the standard adjoint approach. More precisely, we rely on the Lagragian method of Céa [8] for calculating a shape derivative. We recall that we work in the frequency domain. The results obtained here will serve as a basis for the second non-adjoint method we propose in Section 7. 
To simplify the presentation, we compute the shape derivative of the dynamic compliance for one single excitation frequency $\omega$, i.e.

$$
J_{\omega}(\Omega)=\int_{\Gamma_{N}}\left(f_{\operatorname{Im}} \cdot u_{\operatorname{Re}}(\omega)-f_{\operatorname{Re}} \cdot u_{\operatorname{Im}}(\omega)\right) d s .
$$

The shape derivative of (10) is then derived as

$$
J^{\prime}(\Omega)(\theta)=\int_{\omega_{\min }}^{\omega_{\max }} J_{\omega}^{\prime}(\Omega)(\theta) d \omega .
$$

Proposition 4.1. Assume that the damping is not zero, namely $c(x) \geq 0$ and $c \neq 0$. The shape derivative of the single-frequency dynamic compliance is

$$
\begin{gathered}
J_{\omega}^{\prime}(\Omega)(\theta)=\int_{\Gamma}\left(-\omega^{2} \rho\left(u_{\operatorname{Re}} \cdot p_{\operatorname{Re}}+u_{\operatorname{Im}} \cdot p_{\mathrm{Im}}\right)-\omega c\left(u_{\mathrm{Im}} \cdot p_{\operatorname{Re}}-u_{\mathrm{Re}} \cdot p_{\mathrm{Im}}\right)\right. \\
\left.+A e\left(u_{\operatorname{Re}}\right) \cdot e\left(p_{\operatorname{Re}}\right)+A e\left(u_{\mathrm{Im}}\right) \cdot e\left(p_{\mathrm{Im}}\right)\right) \theta \cdot n d s,
\end{gathered}
$$

where $\left(p_{\mathrm{Re}}, p_{\mathrm{Im}}\right)$ is the adjoint state, solution of

$$
\left\{\begin{aligned}
-\omega^{2} \rho p_{\operatorname{Re}}+\omega c p_{\operatorname{Im}}-\operatorname{div}\left(A e\left(p_{\operatorname{Re}}\right)\right) & =0 & & \text { in } \Omega, \\
p_{\operatorname{Re}} & =0 & & \text { on } \Gamma_{D}, \\
\left(A e\left(p_{\operatorname{Re}}\right)\right) n & =-f_{\operatorname{Im}} & & \text { on } \Gamma_{N}, \\
\left.A e\left(p_{\operatorname{Re}}\right)\right) n & =0 & & \text { on } \Gamma,
\end{aligned}\right.
$$

and

$$
\left\{\begin{aligned}
-\omega^{2} \rho p_{\operatorname{Im}}-\omega c p_{\operatorname{Re}}-\operatorname{div}\left(A e\left(p_{\operatorname{Im}}\right)\right) & =0 & & \text { in } \Omega, \\
p_{\operatorname{Im}} & =0 & & \text { on } \Gamma_{D}, \\
\left(A e\left(p_{\operatorname{Im}}\right)\right) n & =f_{\operatorname{Re}} & & \text { on } \Gamma_{N}, \\
\left(A e\left(p_{\operatorname{Im}}\right)\right) n & =0 & & \text { on } \Gamma .
\end{aligned}\right.
$$

Remark 4.1. The numerical computation of the dynamic compliance (10) and its shape derivative (14) requires to compute the state $\left(u_{\operatorname{Re}}, u_{\operatorname{Im}}\right)$ and the adjoint $\left(p_{\operatorname{Re}}, p_{\operatorname{Im}}\right)$ for every discrete frequency $\omega$ in the frequency range $\left[\omega_{\min }, \omega_{\max }\right]$. This is too costly for most real-life industrial problems. For this reason, modal basis approaches are usually prefered, as we shall explain in the next section.

Proof. Since the Dirichlet boundary $\Gamma_{D}$ is fixed, we can introduce a Sobolev space $V$, defined by

$$
V=\left\{v \in H^{1}\left(\mathbb{R}^{d}\right)^{d} \text { such that } v=0 \text { on } \Gamma_{D}\right\},
$$

which is independent of the choice of the shape $\Omega$. Following the method of Céa, we define a Lagrangian which is the sum of the objective function and of the variational formulation of (4),

$$
\begin{aligned}
\mathcal{L}(\Omega, v, q)= & J_{\omega}\left(v_{\mathrm{Re}}, v_{\mathrm{Im}}\right) \\
& +\int_{\Omega}\left(-\omega^{2} \rho v_{\mathrm{Re}} \cdot q_{\mathrm{Re}}-\omega c v_{\mathrm{Im}} \cdot q_{\mathrm{Re}}+A e\left(v_{\mathrm{Re}}\right) \cdot e\left(q_{\mathrm{Re}}\right)\right) d x-\int_{\Gamma_{N}} f_{\mathrm{Re}} \cdot q_{\mathrm{Re}} d s \\
& +\int_{\Omega}\left(-\omega^{2} \rho v_{\mathrm{Im}} \cdot q_{\mathrm{Im}}+\omega c v_{\mathrm{Re}} \cdot q_{\mathrm{Im}}+A e\left(v_{\mathrm{Im}}\right) \cdot e\left(q_{\mathrm{Im}}\right)\right) d x-\int_{\Gamma_{N}} f_{\mathrm{Im}} \cdot q_{\mathrm{Im}} d s
\end{aligned}
$$

where $v_{\mathrm{Re}}, v_{\mathrm{Im}} \in V$ plays the role of the state, $q_{\mathrm{Re}}, q_{\mathrm{Im}} \in V$ are the adjoints or Lagrange multipliers for the state equation and, for the dynamic compliance,

$$
J_{\omega}\left(v_{\mathrm{Re}}, v_{\operatorname{Im}}\right)=\int_{\Gamma_{N}}\left(f_{\mathrm{Im}} \cdot v_{\mathrm{Re}}-f_{\mathrm{Re}} \cdot v_{\operatorname{Im}}\right) d s .
$$


We fix a domain $\Omega$ and consider the optimality conditions for the Lagrangian $\mathcal{L}$ at the optimal point $\left(\Omega, v^{*}, q^{*}\right)$. Obviously, the conditions

$$
\left\langle\frac{\partial \mathcal{L}}{\partial q_{\mathrm{Re}}}\left(\Omega, v^{*}, q^{*}\right), \phi\right\rangle=0, \quad\left\langle\frac{\partial \mathcal{L}}{\partial q_{\mathrm{Im}}}\left(\Omega, v^{*}, q^{*}\right), \phi\right\rangle=0,
$$

for a smooth test function $\phi \in V$, reveal that $\left(v_{\mathrm{Re}}^{*}, v_{\mathrm{Im}}^{*}\right)=\left(u_{\mathrm{Re}}, u_{\mathrm{Im}}\right)$ is the unique solution of the variational formulation for the coupled system of equations (5) and (6).

The partial derivative of $\mathcal{L}$ with respect to $v_{\operatorname{Re}}$, in the direction of a test function $\phi \in V$, at the optimal point, reads

$$
\begin{aligned}
\left\langle\frac{\partial \mathcal{L}}{\partial v_{\operatorname{Re}}}\left(\Omega, v^{*}, q^{*}\right), \phi\right\rangle & =\left\langle\frac{\partial J_{\omega}}{\partial v_{\operatorname{Re}}}\left(v_{\mathrm{Re}}^{*}, v_{\mathrm{Im}}^{*}\right), \phi\right\rangle+\int_{\Omega}\left(-\omega^{2} \rho \phi \cdot q_{\mathrm{Re}}^{*}+\omega c \phi \cdot q_{\mathrm{Im}}^{*}+A e(\phi) \cdot e\left(q_{\mathrm{Re}}^{*}\right)\right) d x \\
& =\int_{\Gamma_{N}} f_{\mathrm{Im}} \cdot \phi d s+\int_{\Omega}\left(-\omega^{2} \rho \phi \cdot q_{\mathrm{Re}}^{*}+\omega c \phi \cdot q_{\mathrm{Im}}^{*}+A e(\phi) \cdot e\left(q_{\mathrm{Re}}^{*}\right)\right) d x .
\end{aligned}
$$

A similar formula can be obtained for the partial derivative of $\mathcal{L}$ with respect to $v_{\mathrm{Im}}$. Setting (18) equal to zero (as well as the other formula), we get that $\left(q_{\mathrm{Re}}^{*}, q_{\mathrm{Im}}^{*}\right)=\left(p_{\mathrm{Re}}, p_{\mathrm{Im}}\right)$ is the solution of the adjoint system (15) and (16).

Finally, the shape derivative of the objective function is just the shape partial derivative of the Lagrangian at the optimal point $\left(v^{*}, q^{*}\right)$ [1], [8] (this requires that the solution $v^{*}$ of the coupled system of equations (5) and (6) is shape differentiable, which is a classical result). In other words, the shape derivative of $J_{\omega}$ reads

$$
\begin{aligned}
J_{\omega}^{\prime}(\Omega)(\theta)=\mathcal{L}^{\prime}\left(\Omega, v^{*}, q^{*}\right)(\theta)=\int_{\Gamma}(\theta \cdot n)( & -\omega^{2} \rho u_{\mathrm{Re}} \cdot q_{\mathrm{Re}}^{*}-\omega c u_{\mathrm{Im}} \cdot q_{\mathrm{Re}}^{*}+A e\left(u_{\mathrm{Re}}\right) \cdot e\left(q_{\mathrm{Re}}^{*}\right) \\
& \left.-\omega^{2} \rho u_{\mathrm{Im}} \cdot q_{\mathrm{Im}}^{*}+\omega c u_{\mathrm{Re}} \cdot q_{\mathrm{Im}}^{*}+A e\left(u_{\mathrm{Im}}\right) \cdot e\left(q_{\mathrm{Im}}^{*}\right)\right) d s,
\end{aligned}
$$

which is nothing but (14).

Remark 4.2. Proposition 4.1 still holds true when there is no damping, $c=0$, provided that $\omega$ is not a resonance frequency (corresponding to an eigenvalue of the problem). In such an undamped case, the problem is self-adjoint since $\left(p_{\operatorname{Re}}, p_{\mathrm{Im}}\right)=\left(-u_{\mathrm{Im}}, u_{\mathrm{Re}}\right)$. In general, for $c \neq 0$, the optimization of the dynamic compliance is not a self-adjoint problem, meaning that the adjoint $\left(p_{\mathrm{Re}}, p_{\mathrm{Im}}\right)$ is not a simple combination of the state $\left(u_{\mathrm{Re}}, u_{\mathrm{Im}}\right)$. There are however a few cases where it is indeed self-adjoint. In case $f_{\mathrm{Im}}=0$, by comparison we find $p_{\mathrm{Re}}=u_{\mathrm{Im}}$ and $p_{\mathrm{Im}}=u_{\mathrm{Re}}$. Therefore the shape derivative (14) reads

$$
J_{\omega}^{\prime}(\Omega)(\theta)=\int_{\Gamma}\left(-2 \omega^{2} \rho u_{\mathrm{Re}} \cdot u_{\mathrm{Im}}-\omega c\left(\left|u_{\mathrm{Im}}\right|^{2}-\left|u_{\mathrm{Re}}\right|^{2}\right)+2 A e\left(u_{\mathrm{Re}}\right) \cdot e\left(u_{\mathrm{Im}}\right)\right) \theta \cdot n d s .
$$

In case $f_{\mathrm{Re}}=0$, by comparison we find $p_{\mathrm{Re}}=-u_{\mathrm{Im}}, p_{\mathrm{Im}}=-u_{\mathrm{Re}}$ and the shape derivative (14) reads

$$
J_{\omega}^{\prime}(\Omega)(\theta)=\int_{\Gamma}\left(2 \omega^{2} \rho u_{\mathrm{Re}} \cdot u_{\mathrm{Im}}+\omega c\left(\left|u_{\mathrm{Im}}\right|^{2}-\left|u_{\mathrm{Re}}\right|^{2}\right)-2 A e\left(u_{\mathrm{Re}}\right) \cdot e\left(u_{\mathrm{Im}}\right)\right) \theta \cdot n d s .
$$

Remark 4.3. In the spirit of Remark 2.3, in matrix notation, the adjoint equation can be written

$$
\left[\begin{array}{cc}
K-\omega^{2} M & -\omega C \\
\omega C & K-\omega^{2} M
\end{array}\right]\left[\begin{array}{c}
p_{\operatorname{Im}} \\
p_{\operatorname{Re}}
\end{array}\right]=\left[\begin{array}{c}
f_{\mathrm{Re}} \\
-f_{\operatorname{Im}}
\end{array}\right]
$$

or, equivalently, $\overline{S(\omega)} P=i F$ with the notation $P=p_{\operatorname{Re}}+i p_{\operatorname{Im}}$ and the dynamic stiffness matrix $S(\omega)=K+i \omega C-\omega^{2} M$. The matrix in (21) is the same as in (7) for the direct problem so its factorization can be kept in order to minimize the overhead of solving for an adjoint. However, there are as many linear systems to solve than frequencies $\omega$ in the discretization of the dynamic compliance. 


\section{Modal analysis using an adjoint method}

The modal decomposition allows to solve large-scale dynamics problems in reasonable time. In frequency response problems, the great majority of publications and commercial softwares use a modal analysis, coupled with an adjoint state for every mode considered. For reasons of completeness, although classical in the literature, we present here the detailed shape derivation using this approach.

\subsection{Modal decomposition}

We introduce the modal basis for the elasticity problem (4) without damping, i.e. $c=0$. The eigenvalues are the squares of the eigenfrequencies $\omega_{j}>0, j \geq 1$, labelled by increasing order with repeated multiplicities. The eigenmodes $r_{j}$ are real vector-valued functions which satisfy

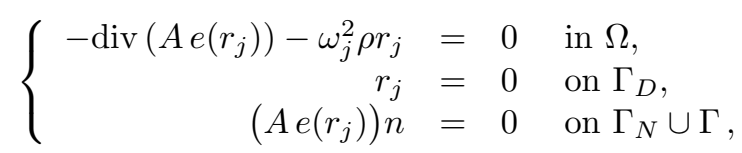

and are normalized by

$$
\int_{\Omega} \rho r_{j} \cdot r_{j} d x=1
$$

The damped elasticity equation (4) can not be diagonalized by these eigenmodes in full generality because the damping term $c(x)$ is not a spectral combination of the inertia term $\rho(x)$ and of the elasticity operator $\operatorname{div}(A e(\cdot))$. However, in engineering practice, the damping term is often assumed to be diagonalizable. We adhere to this setting and define a damping linear operator $C$ which is somehow a linear combination of the inertia term and of the elasticity operator and will be defined more precisely later (see (26) below) by its spectral decomposition. In other words, we replace (4) by

$$
\left\{\begin{aligned}
-\omega^{2} \rho U+i \omega C(U)-\operatorname{div}(A e(U)) & =0 & & \text { in } \Omega, \\
U & =0 & & \text { on } \Gamma_{D}, \\
(A e(U)) n & =F & & \text { on } \Gamma_{N}, \\
(A e(U)) n & =0 & & \text { on } \Gamma .
\end{aligned}\right.
$$

The complex amplitude $U$ is decomposed in the basis formed by the eigenvectors $r_{j}$, such that

$$
U(x)=\sum_{j=1}^{\infty} a^{j} r_{j}(x) \quad \text { with } \quad a^{j}=a_{\mathrm{Re}}^{j}+i a_{\mathrm{Im}}^{j} \quad \text { and } \quad a_{\mathrm{Re}}^{j}, a_{\mathrm{Im}}^{j} \in \mathbb{R} .
$$

To compute the coordinates $a^{j}$, we substitute (25) in (24) and take its variational formulation with the test function $r_{k}$ (the $k$-th eigenfunction). It leads to

$\sum_{j=1}^{\infty} a^{j}\left(-\omega^{2} \int_{\Omega} \rho r_{j} \cdot r_{k} d x+i \omega \int_{\Omega} C\left(r_{j}\right) \cdot r_{k} d x+\int_{\Omega} A e\left(r_{j}\right) \cdot e\left(r_{k}\right) d x\right)=\int_{\Gamma_{N}} f_{\mathrm{Re}} \cdot r_{k} d s+i \int_{\Gamma_{N}} f_{\mathrm{Im}} \cdot r_{k} d s$.

By using the orthogonality property of the eigenfunctions, we have, for $j \neq k$,

$$
\int_{\Omega} \rho r_{j} \cdot r_{k} d x=0 \quad \text { and } \quad \int_{\Omega} A e\left(r_{j}\right) \cdot e\left(r_{k}\right) d x=0
$$

and for $j=k$

$$
\int_{\Omega} \rho r_{k} \cdot r_{k} d x=M_{k k}>0 \quad \text { and } \quad \int_{\Omega} A e\left(r_{k}\right) \cdot e\left(r_{k}\right) d x=K_{k k}>0
$$


We now make precise our definition of the assumed damping operator $C$ which is diagonal in the same basis with

$$
\int_{\Omega} C\left(r_{j}\right) \cdot r_{k} d x= \begin{cases}0 & \text { if } j \neq k \\ C_{k k}=2 M_{k k} \omega_{k} \xi_{k} & \text { if } j=k\end{cases}
$$

where $\xi_{k}>0$ denotes the damping ratio for the eigenmode $k$. We thus deduce

$$
a^{k}=\frac{<f_{\mathrm{Re}}, r_{k}>_{\Gamma_{N}}+i<f_{\mathrm{Im}}, r_{k}>_{\Gamma_{N}}}{\left(K_{k k}-\omega^{2} M_{k k}\right)+i \omega C_{k k}}
$$

with the following notation

$$
<f, r_{k}>_{\Gamma_{N}}=\int_{\Gamma_{N}} f \cdot r_{k} d x .
$$

Now, our normalization assumption implies that $M_{k k}=1$ and $K_{k k}=\omega_{k}^{2}$, which yields

$$
\begin{aligned}
a^{k} & =\frac{<f_{\mathrm{Re}}, r_{k}>_{\Gamma_{N}}+i<f_{\operatorname{Im}}, r_{k}>_{\Gamma_{N}}}{\left(\omega_{k}^{2}-\omega^{2}\right)+i\left(2 \omega \omega_{k} \xi_{k}\right)} \\
& =\frac{\left(<f_{\mathrm{Re}}, r_{k}>_{\Gamma_{N}}+i<f_{\operatorname{Im}}, r_{k}>_{\Gamma_{N}}\right)\left(\left(\omega_{k}^{2}-\omega^{2}\right)-i\left(2 \omega \omega_{k} \xi_{k}\right)\right)}{\left(\omega_{k}^{2}-\omega^{2}\right)^{2}+\left(2 \omega \omega_{k} \xi_{k}\right)^{2}} .
\end{aligned}
$$

Note that $a^{k}$ is always finite because its denominator cannot vanish, even if $\omega=\omega_{k}$, since $\xi_{k} \neq 0$ by assumption. Eventually, the complex amplitude function $U=u_{\operatorname{Re}}+i u_{\operatorname{Im}}$ is obtained as an explicit function of the full set of eigenfrequencies and eigenmodes $\left\{\omega_{j}, r_{j}\right\}_{j=1}^{\infty}$

$$
u_{\mathrm{Re}}=\sum_{j=1}^{\infty}\left(\frac{<f_{\mathrm{Re}}, r_{j}>_{\Gamma_{N}}\left(\omega_{j}^{2}-\omega^{2}\right)+<f_{\mathrm{Im}}, r_{j}>_{\Gamma_{N}} 2 \omega \omega_{j} \xi_{j}}{\left(\omega_{j}^{2}-\omega^{2}\right)^{2}+\left(2 \omega \omega_{j} \xi_{j}\right)^{2}}\right) r_{j}
$$

and

$$
u_{\mathrm{Im}}=\sum_{j=1}^{\infty}\left(\frac{<f_{\mathrm{Im}}, r_{j}>_{\Gamma_{N}}\left(\omega_{j}^{2}-\omega^{2}\right)-<f_{\mathrm{Re}}, r_{j}>_{\Gamma_{N}} 2 \omega \omega_{j} \xi_{j}}{\left(\omega_{j}^{2}-\omega^{2}\right)^{2}+\left(2 \omega \omega_{j} \xi_{j}\right)^{2}}\right) r_{j} .
$$

Remark 5.1. In the above analysis, the damping coefficients have been modelled by (26), i.e., $C_{k k}=2 M_{k k} \omega_{k} \xi_{k}$. Although this is a practical and popular assumption in engineering practice [10], there are different models which could equally be considered. For example, another classical model amounts to assuming that the damping matrix is proportional to a linear combination of the mass and stiffness matrices, i.e. $C=\alpha M+\beta K$, with coefficients $\alpha, \beta>0$, independent of the eigenfrequencies $\omega_{k}$.

Remark 5.2. Of course, in numerical practice the series in formulas (28) and (29) are truncated to a finite number of modes $j \leq n_{\bmod }$ in order to obtain a computable approximation of $U$. $A$ discussion of the numerical cost is given later in Section 8.

\subsection{Shape derivative}

We now give a different formula for the shape derivative of the dynamic compliance, based on the modal decomposition of the previous subsection. The main idea is to replace the objective function $J(\Omega)$, which depends on the solution $U$ of the damped wave equation (4), by the same objective function $J^{\bmod }(\Omega)$ which is written in terms of the modal decomposition (28) and (29) of $U$ and thus is a function of the eigenvalues and eigenmodes. To simplify the presentation, we again consider the single-frequency dynamic compliance

$$
J_{\omega}(\Omega)=\int_{\Gamma_{N}}\left(f_{\operatorname{Im}} \cdot u_{\operatorname{Re}}(\omega)-f_{\operatorname{Re}} \cdot u_{\operatorname{Im}}(\omega)\right) d s .
$$


Proposition 5.1. Assume that the damping is not zero and is modelled by (26), with $\xi_{j}>0$ for all modes. Assume that all eigenfrequencies $\omega_{j}$ are simple. The shape derivative of the single-frequency dynamic compliance is

$$
J_{\omega}^{\prime}(\Omega)(\theta)=\int_{\Gamma} \theta \cdot n \sum_{j=1}^{\infty}\left(A e\left(r_{j}\right) \cdot e\left(q_{j}\right)-\omega_{j}^{2} \rho r_{j} \cdot q_{j}+\mu_{j} \rho\left|r_{j}\right|^{2}\right) d s .
$$

where $q_{j}$ is the adjoint state, solution of the adjoint equation (33), and $\mu_{j}$ is a Lagrange multiplier defined by (38).

Remark 5.3. We emphasize that Proposition 5.1 is valid only if the eigenfrequencies $\omega_{j}$ and eigenmodes $r_{j}$ are shape differentiable. This is usually achieved by assuming that all eigenvalues are simple (multiplicity equal to one). Therefore, the analysis presented here is valid only if multiple eigenvalues are not present.

Remark 5.4. Formulas (14) and (30) for the shape derivative of the dynamic compliance should be equivalent although it is not easy to check. Of course, in the context of Proposition 5.1, we made a different spectral assumption on the damping than in Proposition 4.1, so this comparison has to be made only when there is no damping $c=0$.

Remark 5.5. The shape derivative of the dynamic compliance (10) is recovered from (30) by integrating with respect to $\omega$, namely

$$
J^{\prime}(\Omega)(\theta)=\int_{\omega_{\min }}^{\omega_{\max }} J_{\omega}^{\prime}(\Omega)(\theta) d \omega=\int_{\Gamma} \theta \cdot n \sum_{j=1}^{\infty}\left(A e\left(r_{j}\right) \cdot e\left(\widetilde{q_{j}}\right)-\omega_{j}^{2} \rho r_{j} \cdot \widetilde{q_{j}}+\widetilde{\mu_{j}} \rho\left|r_{j}\right|^{2}\right) d s,
$$

where

$$
\widetilde{q_{j}}=\int_{\omega_{\min }}^{\omega_{\max }} q_{j} d \omega \quad \text { and } \quad \widetilde{\mu_{j}}=\int_{\omega_{\min }}^{\omega_{\max }} \mu_{j} d \omega .
$$

Of course, the adjoints $q_{j}$ depend on the excitation frequency $\omega$. However, by inspecting the adjoint equation (33), the excitation frequency appears only in its right hand side. In other words, for different excitation frequencies $\omega$, the adjoints $q_{j}(\omega)$ share the same differential operator or rigidity matrix. Therefore, the averaged adjoint $\widetilde{q_{j}}$ can be computed as the solution of the adjoint equation (33), where the right hand side is also averaged with respect to $\omega$, and only one adjoint equation per eigenfrequency has to be solved (whatever the number of excitation frequencies). In numerical practice, only a finite number $n_{\bmod }$ of eigenmodes is used to approximate the shape derivative (30). Therefore, from a computation point of view, formula (30) is superior to the previous formula (14) since it requires less adjoints because the number of eigenmodes $n_{\bmod }$ is usually much smaller than the number of discretized excitation frequencies in the definition of the dynamic compliance. Neverteless, the overall CPU cost of the method of Proposition 5.1 is still quite expensive. See Section 8 for more details.

Proof. Any objective function $J(\Omega)=J\left(u_{\mathrm{Re}}(\Omega), u_{\mathrm{Im}}(\Omega)\right)$, which is defined in terms of the solution $\left(u_{\mathrm{Re}}, u_{\mathrm{Im}}\right)$ of $(5),(6)$, can also be considered as a function of the full set of eigenfrequencies and eigenmodes $\left\{\omega_{j}, r_{j}\right\}_{j=1}^{\infty}$ by virtue of the modal decomposition (28) and (29). We denote by $J^{\bmod }$ this function, defined by

$$
J\left(u_{\mathrm{Re}}, u_{\mathrm{Im}}\right)=J^{\bmod }\left(\left\{\omega_{j}, r_{j}\right\}_{j=1}^{\infty}\right) .
$$

To compute the shape derivative of $J_{\omega}(\Omega)$, we use once more the method of Céa but applied to the function $J_{\omega}^{\bmod }$, considering the spectral equation (22) as a constraint, instead of the elasticity 
problem (4). In other words, we introduce the following Lagrangian

$$
\begin{aligned}
\mathcal{L}\left(\Omega, \widehat{r}_{j}, \widehat{q}_{j}, \widehat{\omega}_{j}, \widehat{\mu}_{j}\right)= & J_{\omega}^{\bmod }\left(\left\{\widehat{\omega}_{j}, \widehat{r}_{j}\right\}_{j=1}^{\infty}\right) \\
& +\sum_{j=1}^{\infty} \int_{\Omega}\left(A e\left(\widehat{r}_{j}\right) \cdot e\left(\widehat{q}_{j}\right)-\widehat{\omega}_{j}^{2} \rho \widehat{r}_{j} \cdot \widehat{q}_{j}\right) d x+\sum_{j=1}^{\infty} \widehat{\mu}_{j}\left(\int_{\Omega} \rho\left|\widehat{r}_{j}\right|^{2} d x-1\right),
\end{aligned}
$$

where $\widehat{r}_{j}, \widehat{q}_{j} \in V$ and $\widehat{\omega}_{j}, \widehat{\mu}_{j} \in \mathbb{R} \forall j=1, \ldots, \infty$. The variables $\widehat{\omega}_{j}, \widehat{r}_{j}$ will be exactly the eigenfrequencies and eigenmodes $\omega_{j}, r_{j}$ at optimality, while $\mu_{j}, q_{j}$ are the corresponding adjoint variables or Lagrange multipliers. For a given domain $\Omega$, setting the partial derivative of $\mathcal{L}$ with respect to $q_{j}$ equal to zero, at the optimal point $\left(r_{j}, q_{j}, \omega_{j}, \mu_{j}\right)$, yields

$$
\left\langle\frac{\partial \mathcal{L}}{\partial q_{j}}\left(\Omega, r_{j}, q_{j}, \omega_{j}, \mu_{j}\right), \phi\right\rangle=0 \quad \text { for any } \phi \in V,
$$

which is nothing but the variational formulation of the eigenvalue problem (22). It has non trivial solutions if and only if $\omega_{j}$ is indeed an eigenfrequency. Then, by simplicity of the eigenvalue, $r_{j}$ is proportional to the corresponding eigenmode. Setting the partial derivative of $\mathcal{L}$ with respect to $\mu_{j}$ at the optimal point equal to zero, yields

$$
\int_{\Omega} \rho\left|r_{j}\right|^{2} d x=1
$$

which is the normalization condition for the eigenfunction, thus we deduce that $r_{j}$ is the unique (up to a possible sign change) normalized eigenmode.

We now turn to the partial derivatives of $\mathcal{L}$ which leads to the adjoint problems. Since, for the dynamic compliance,

$$
\left\langle\frac{\partial J_{\omega}^{\bmod }}{\partial r_{j}}, \phi\right\rangle=\frac{\partial J_{\omega}}{\partial u_{\mathrm{Re}}}\left\langle\frac{\partial u_{\mathrm{Re}}}{\partial r_{j}}, \phi\right\rangle+\frac{\partial J_{\omega}}{\partial u_{\mathrm{Im}}}\left\langle\frac{\partial u_{\mathrm{Im}}}{\partial r_{j}}, \phi\right\rangle=\int_{\Gamma_{N}}\left(\left\langle\frac{\partial u_{\mathrm{Re}}}{\partial r_{j}}, \phi\right\rangle f_{\mathrm{Im}}-\left\langle\frac{\partial u_{\mathrm{Im}}}{\partial r_{j}}, \phi\right\rangle f_{\mathrm{Re}}\right) d s,
$$

setting to zero the partial derivative with respect to $r_{j}$ of the Lagrangian (31) yields the variational formulation of the following adjoint equation

$$
\left\{\begin{aligned}
-\operatorname{div}\left(A e\left(q_{j}\right)\right)-\omega_{j}^{2} \rho q_{j} & =-2 \mu_{j} \rho r_{j} & & \text { in } \Omega, \\
q_{j} & =0 & & \text { on } \Gamma_{D}, \\
\left(A e\left(q_{j}\right)\right) n & =-\frac{\partial J_{\omega}^{m o d}}{\partial r_{j}}\left(u_{\operatorname{Re}}\left(\omega_{j}, r_{j}\right), u_{\operatorname{Im}}\left(\omega_{j}, r_{j}\right)\right) & & \text { on } \Gamma_{N} \\
\left(A e\left(q_{j}\right)\right) n & =0 & & \text { on } \Gamma
\end{aligned}\right.
$$

The Neumann boundary data $\frac{\partial J_{\omega}^{\text {mod }}}{\partial r_{j}}\left(u_{\mathrm{Re}}\left(\omega_{j}, r_{j}\right), u_{\mathrm{Im}}\left(\omega_{j}, r_{j}\right)\right)$ in the adjoint equation (33) can be made explicit by recalling the modal decomposition. Based on (28) we define

$$
u_{\operatorname{Re}}\left(\left\{\widehat{\omega}_{j}, \widehat{r}_{j}\right\}_{j=1}^{\infty}\right)=\sum_{j=1}^{\infty} \alpha_{\operatorname{Re}}\left(\widehat{\omega}_{j}, \widehat{r}_{j}\right) \widehat{r}_{j}
$$

with $\quad \alpha_{\operatorname{Re}}\left(\widehat{\omega}_{j}, \widehat{r}_{j}\right)=\frac{<f_{\operatorname{Re}}, \widehat{r}_{j}>_{\Gamma_{N}}\left(\widehat{\omega}_{j}^{2}-\omega^{2}\right)+<f_{\operatorname{Im}}, \widehat{r}_{j}>_{\Gamma_{N}} 2 \omega \widehat{\omega}_{j} \xi_{j}}{\left(\widehat{\omega}_{j}^{2}-\omega^{2}\right)^{2}+\left(2 \omega \widehat{\omega}_{j} \xi_{j}\right)^{2}}$,

and similarly from $(29)$

$$
\begin{gathered}
u_{\operatorname{Im}}\left(\left\{\widehat{\omega}_{j}, \widehat{r}_{j}\right\}_{j=1}^{\infty}\right)=\sum_{j=1}^{\infty} \alpha_{\operatorname{Im}}\left(\widehat{\omega}_{j}, \widehat{r}_{j}\right) \widehat{r}_{j} \\
\text { with } \quad \alpha_{\operatorname{Im}}\left(\widehat{\omega}_{j}, \widehat{r}_{j}\right)=\frac{<f_{\operatorname{Im}}, \widehat{r}_{j}>_{\Gamma_{N}}\left(\widehat{\omega}_{j}^{2}-\omega^{2}\right)-<f_{\mathrm{Re}}, \widehat{r}_{j}>_{\Gamma_{N}} 2 \omega \widehat{\omega}_{j} \xi_{j}}{\left(\widehat{\omega}_{j}^{2}-\omega^{2}\right)^{2}+\left(2 \omega \widehat{\omega}_{j} \xi_{j}\right)^{2}} .
\end{gathered}
$$


Thus, we deduce

$$
\begin{aligned}
\left\langle\frac{\partial u_{\mathrm{Re}}}{\partial r_{j}}, \phi_{j}\right\rangle= & \frac{<f_{\operatorname{Re}}, \phi_{j}>_{\Gamma_{N}}\left(\widehat{\omega}_{j}^{2}-\omega^{2}\right)+<f_{\mathrm{Im}}, \phi_{j}>_{\Gamma_{N}} 2 \omega \widehat{\omega}_{j} \xi_{j}}{\left(\widehat{\omega}_{j}^{2}-\omega^{2}\right)^{2}+\left(2 \omega \widehat{\omega}_{j} \xi_{j}\right)^{2}} \widehat{r}_{j} \\
& +\frac{<f_{\operatorname{Re}}, \widehat{r}_{j}>_{\Gamma_{N}}\left(\widehat{\omega}_{j}^{2}-\omega^{2}\right)+<f_{\mathrm{Im}}, \widehat{r}_{j}>_{\Gamma_{N}} 2 \omega \widehat{\omega}_{j} \xi_{j}}{\left(\widehat{\omega}_{j}^{2}-\omega^{2}\right)^{2}+\left(2 \omega \widehat{\omega}_{j} \xi_{j}\right)^{2}} \phi_{j}
\end{aligned}
$$

and

$$
\begin{aligned}
\left\langle\frac{\partial u_{\operatorname{Im}}}{\partial r_{j}}, \phi_{j}\right\rangle= & \frac{<f_{\operatorname{Im}}, \phi_{j}>_{\Gamma_{N}}\left(\widehat{\omega}_{j}^{2}-\omega^{2}\right)-<f_{\mathrm{Re}}, \phi_{j}>_{\Gamma_{N}} 2 \omega \widehat{\omega}_{j} \xi_{j}}{\left(\widehat{\omega}_{j}^{2}-\omega^{2}\right)^{2}+\left(2 \omega \widehat{\omega}_{j} \xi_{j}\right)^{2}} \widehat{r}_{j} \\
& +\frac{<f_{\operatorname{Im}}, \widehat{r}_{j}>_{\Gamma_{N}}\left(\widehat{\omega}_{j}^{2}-\omega^{2}\right)-<f_{\mathrm{Re}}, \widehat{r}_{j}>_{\Gamma_{N}} 2 \omega \widehat{\omega}_{j} \xi_{j}}{\left(\widehat{\omega}_{j}^{2}-\omega^{2}\right)^{2}+\left(2 \omega \widehat{\omega}_{j} \xi_{j}\right)^{2}} \phi_{j} .
\end{aligned}
$$

Notice that taking the test function $\phi_{j}=\widehat{r}_{j}$ gives the simplified formula

$$
\left\langle\frac{\partial u_{\operatorname{Re}}}{\partial r_{j}}, \widehat{r}_{j}\right\rangle=2 \alpha_{\operatorname{Re}}\left(\widehat{\omega}_{j}, \widehat{r}_{j}\right) \widehat{r}_{j} \quad \text { and } \quad\left\langle\frac{\partial u_{\operatorname{Im}}}{\partial r_{j}}, \widehat{r}_{j}\right\rangle=2 \alpha_{\operatorname{Im}}\left(\widehat{\omega}_{j}, \widehat{r}_{j}\right) \widehat{r}_{j} .
$$

Plugging these derivatives, computed at the optimal point $\left(r_{j}, q_{j}, \omega_{j}, \mu_{j}\right)$, in $(32)$ and in the variational formulation of (33) makes it completely explicit, up to the knowledge of $\mu_{j}$.

To find the optimal value $\mu_{j}$ we remark that the differential operator in (33) has a kernel which is nothing but the eigenmode $r_{j}$. Therefore, multiplying equation (33) by $r_{j}$ and integrating by parts, we find that the value of $\mu_{j}$ exactly balances the non-homogeneous Neumann boundary data

$$
\mu_{j}=-\int_{\Gamma_{N}}\left(\alpha_{\operatorname{Re}}\left(\omega_{j}, r_{j}\right) f_{\operatorname{Im}} \cdot r_{j}-\alpha_{\operatorname{Im}}\left(\omega_{j}, r_{j}\right) f_{\operatorname{Re}} \cdot r_{j}\right) d s
$$

It remains to compute the partial derivative of $\mathcal{L}$ with respect to $\omega_{j}$, which will give a normalization condition ensuring the uniqueness of the adjoint $q_{j}$ (see Remark 5.6 below). Setting this partial derivative at the optimal point equal to zero, we obtain:

$$
\frac{\partial \mathcal{L}}{\partial \omega_{j}}\left(\Omega, r_{j}, q_{j}, \omega_{j}, \mu_{j}\right)=\frac{\partial J_{\omega}^{\text {mod }}}{\partial \omega_{j}}\left(u_{\operatorname{Re}}\left(\omega_{j}, r_{j}\right), u_{\operatorname{Im}}\left(\omega_{j}, r_{j}\right)\right)-\int_{\Omega} 2 \omega_{j} \rho r_{j} \cdot q_{j} d x=0 .
$$

For the dynamic compliance the term $\frac{\partial J_{\omega}^{\text {mod }}}{\partial \omega_{j}}\left(u_{\mathrm{Re}}\left(\omega_{j}, r_{j}\right), u_{\operatorname{Im}}\left(\omega_{j}, r_{j}\right)\right)$ reads:

$$
\begin{aligned}
\frac{\partial J_{\omega}^{\text {mod }}}{\partial \omega_{j}}\left(u_{\mathrm{Re}}\left(\omega_{j}, r_{j}\right), u_{\operatorname{Im}}\left(\omega_{j}, r_{j}\right)\right) & =\frac{\partial J_{\omega}^{\text {mod }}}{\partial u_{\mathrm{Re}}} \frac{\partial u_{\mathrm{Re}}}{\partial \omega_{j}}\left(\omega_{j}, r_{j}\right)+\frac{\partial J_{\omega}^{\text {mod }}}{\partial u_{\mathrm{Im}}} \frac{\partial u_{\mathrm{Im}}}{\partial \omega_{j}}\left(\omega_{j}, r_{j}\right) \\
& =\int_{\Gamma_{N}}\left(f_{\operatorname{Im}} \cdot \frac{\partial u_{\mathrm{Re}}}{\partial \omega_{j}}\left(\omega_{j}, r_{j}\right)-f_{\operatorname{Re}} \cdot \frac{\partial u_{\mathrm{Im}}}{\partial \omega_{j}}\left(\omega_{j}, r_{j}\right)\right) d s
\end{aligned}
$$

From the modal decomposition of $u_{\mathrm{Re}}$ and $u_{\mathrm{Im}}$, after some algebra, we find that:

$$
\frac{\partial u_{\operatorname{Re}}\left(\left\{\widehat{\omega}_{j}, \widehat{r}_{j}\right\}_{j=1}^{\infty}\right)}{\partial \omega_{j}}=\beta_{\operatorname{Re}}\left(\widehat{\omega}_{j}, \widehat{r}_{j}\right) \widehat{r}_{j} \quad \text { and } \quad \frac{\partial u_{\operatorname{Im}}\left(\left\{\widehat{\omega}_{j}, \widehat{r}_{j}\right\}_{j=1}^{\infty}\right)}{\partial \omega_{j}}=\beta_{\operatorname{Im}}\left(\widehat{\omega}_{j}, \widehat{r}_{j}\right) \widehat{r}_{j},
$$

where

$$
\begin{aligned}
\beta_{\operatorname{Re}}\left(\widehat{\omega}_{j}, \widehat{r}_{j}\right)= & \frac{\left(-2 \widehat{\omega}_{j}\left(\widehat{\omega}_{j}^{2}-\omega^{2}\right)^{2}+2 \widehat{\omega}_{j}\left(2 \omega \widehat{\omega}_{j} \xi_{j}\right)^{2}-4 \omega \xi_{j}\left(2 \omega \widehat{\omega}_{j} \xi_{j}\right)\left(\widehat{\omega}_{j}^{2}-\omega^{2}\right)\right)<f_{\operatorname{Re}}, \widehat{r}_{j}>_{\Gamma_{N}}}{\left[\left(\widehat{\omega}_{j}^{2}-\omega^{2}\right)^{2}+\left(2 \omega \widehat{\omega}_{j} \xi_{j}\right)^{2}\right]^{2}} \\
& +\frac{\left(2 \omega \xi_{j}\left(\widehat{\omega}_{j}^{2}-\omega^{2}\right)^{2}-2 \omega \xi_{j}\left(2 \omega \widehat{\omega}_{j} \xi_{j}\right)^{2}-4 \widehat{\omega}_{j}\left(\widehat{\omega}_{j}^{2}-\omega^{2}\right)\left(2 \omega \widehat{\omega}_{j} \xi_{j}\right)\right)<f_{\operatorname{Im}}, \widehat{r}_{j}>_{\Gamma_{N}}}{\left[\left(\widehat{\omega}_{j}^{2}-\omega^{2}\right)^{2}+\left(2 \omega \widehat{\omega}_{j} \xi_{j}\right)^{2}\right]^{2}}
\end{aligned}
$$


and

$$
\begin{aligned}
\beta_{\operatorname{Im}}\left(\widehat{\omega}_{j}, \widehat{r}_{j}\right)= & \frac{\left(-2 \widehat{\omega}_{j}\left(\widehat{\omega}_{j}^{2}-\omega^{2}\right)^{2}+2 \widehat{\omega}_{j}\left(2 \omega \widehat{\omega}_{j} \xi_{j}\right)^{2}-4 \omega \xi_{j}\left(2 \omega \widehat{\omega}_{j} \xi_{j}\right)\left(\widehat{\omega}_{j}^{2}-\omega^{2}\right)\right)<f_{\operatorname{Im}}, \widehat{r}_{j}>_{\Gamma_{N}}}{\left[\left(\widehat{\omega}_{j}^{2}-\omega^{2}\right)^{2}+\left(2 \omega \widehat{\omega}_{j} \xi_{j}\right)^{2}\right]^{2}} \\
& -\frac{\left(2 \omega \xi_{j}\left(\widehat{\omega}_{j}^{2}-\omega^{2}\right)^{2}-2 \omega \xi_{j}\left(2 \omega \widehat{\omega}_{j} \xi_{j}\right)^{2}-4 \widehat{\omega}_{j}\left(\widehat{\omega}_{j}^{2}-\omega^{2}\right)\left(2 \omega \widehat{\omega}_{j} \xi_{j}\right)\right)<f_{\mathrm{Re}}, \widehat{r}_{j}>_{\Gamma_{N}}}{\left[\left(\widehat{\omega}_{j}^{2}-\omega^{2}\right)^{2}+\left(2 \omega \widehat{\omega}_{j} \xi_{j}\right)^{2}\right]^{2}} .
\end{aligned}
$$

Therefore equation (39) turns out to be a normalization condition for $q_{j}$ wich reads

$$
2 \int_{\Omega} \omega_{j} \rho r_{j} \cdot q_{j} d x=\int_{\Gamma_{N}}\left(\beta_{\operatorname{Re}}\left(\omega_{j}, r_{j}\right) f_{\mathrm{Im}} \cdot r_{j}-\beta_{\operatorname{Im}}\left(\omega_{j}, r_{j}\right) f_{\mathrm{Re}} \cdot r_{j}\right) d s
$$

Finally, considering vector fields $\theta$ which vanishes on $\Gamma_{D} \cup \Gamma_{N}$, the shape derivative of the objective function $J_{\omega}(\Omega)$ is equal to the shape derivative of the Lagrangian $\mathcal{L}$ at the optimal point, i.e.

$$
\begin{aligned}
J_{\omega}^{\prime}(\Omega)(\theta) & =J_{\omega}^{\bmod ^{\prime}}\left(u_{\mathrm{Re}}\left(\left\{\omega_{j}, r_{j}\right\}_{j=1}^{\infty}\right), u_{\operatorname{Im}}\left(\left\{\omega_{j}, r_{j}\right\}_{j=1}^{\infty}\right)\right)(\theta) \\
& =\int_{\Gamma} \theta \cdot n\left[\sum_{j=1}^{\infty}\left(A e\left(r_{j}\right) \cdot e\left(q_{j}\right)-\omega_{j}^{2} \rho r_{j} \cdot q_{j}+\mu_{j} \rho\left|r_{j}\right|^{2}\right)\right] d s
\end{aligned}
$$

with the optimal values of variables, as determined above.

Remark 5.6. By the differentiability of simple eigenfunctions [17], we know that there must exist a solution of (33). On the other hand, by looking at the structure of the adjoint equation (33), we now check that it admits a unique solution because of the following reasons. First, note that the operator $P=-\operatorname{div}(A e(\cdot))-\omega_{j}^{2} \rho(\cdot)$ on the left-hand side of (33) has a kernel of dimension 1, generated by $r_{j}$. Therefore, the solution is unique, only up to the addition of a multiple of $r_{j}$. The normalization condition (41) uniquely determines this additive term. Second, since $P$ is a selfadjoint operator with compact resolvent, the existence of a solution is guaranteed if the right-hand side belongs to the range of $P$. A formal computation (working as if $P$ was a finite dimensional operator and ignoring any issue of closedness and compactness of unbounded operators) shows that

$$
\operatorname{Im}(P)=\left(\operatorname{Ker} P^{T}\right)^{\perp}=(\operatorname{Ker} P)^{\perp} .
$$

However, denoting by $L(\phi)$ the linear form in the right hand side of the variational formulation of (33), we have

$$
L(\phi)=-2 \mu_{j} \int_{\Omega} \rho r_{j} \cdot \phi d x-\int_{\Gamma_{N}} \frac{\partial J_{\omega}^{\bmod }\left(\omega_{j}, r_{j}\right)}{\partial r_{j}} \cdot \phi d s
$$

and by (38) we see that $L\left(r_{j}\right)=0$, which means that the right hand side $L$ is orthogonal to $\operatorname{Ker} P$ and thus belongs to $\operatorname{Im} P$. Making this argument rigorous in infinite dimensional spaces is the purpose of the Fredholm alternative, a well-known result [6] that we do not discuss further. In numerical practice, solving equation (33) which has a non-trivial kernel may be delicate. Sometimes it is prefered to solve a regularized equation like

$$
\left\{\begin{aligned}
-\operatorname{div}\left(A e\left(q_{j}^{\varepsilon}\right)\right)-\omega_{j}^{2} \rho q_{j}^{\varepsilon}+\varepsilon\left(\rho r_{j} \otimes r_{j}\right) q_{j}^{\varepsilon} & =-2 \mu_{j} \rho r_{j} & & \text { in } \Omega, \\
q_{j}^{\varepsilon} & =0 & & \text { on } \Gamma_{D}, \\
\left(A e\left(q_{j}^{\varepsilon}\right)\right) n & =-\frac{\partial J_{\omega}^{\text {mod }}}{\partial r_{j}}\left(\omega_{j}, r_{j}\right) & & \text { on } \Gamma_{N}, \\
\left(A e\left(q_{j}^{\varepsilon}\right)\right) n & =0 & & \text { on } \Gamma,
\end{aligned}\right.
$$


where $\varepsilon>0$ is a small positive parameter $(\varepsilon<<1)$ and we have denoted

$$
\left(\rho r_{j} \otimes r_{j}\right) q_{j}^{\varepsilon}=\left(\int_{\Omega} \rho r_{j} \cdot q_{j}^{\varepsilon} d x\right) r_{j} .
$$

Equation (43) has a unique solution. Then, an approximation of the true adjoint state is obtained as $q_{j} \approx q_{j}^{\varepsilon}-\left(\rho r_{j} \otimes r_{j}\right) q_{j}^{\varepsilon}$, in order to satisfy that $q_{j}^{\varepsilon} \perp r_{j}$.

\section{Modal analysis without any adjoint: direct computation of the eigenmodes' derivatives}

In this section we present a first new approach which does not require the computation of any adjoint solutions. As we have seen in Section 5 , in order to calculate the shape derivative for a general objective function of the type

$$
J(\Omega)=J\left(u_{\mathrm{Re}}(\Omega), u_{\mathrm{Im}}(\Omega)\right)=J^{\bmod }\left(\left\{\omega_{j}, r_{j}\right\}_{j=1}^{\infty}\right),
$$

where $\left(\omega_{j}^{2}, r_{j}\right), j \in\{1, \ldots, \infty\}$ are the eigenvalues and eigenmodes, solution of (22), we need to compute an adjoint state for each mode $j$. Using the finite element method to solve the adjoint equation (33), we observe that the stiffness matrix needs to be recalculated for every eigenmode, which augments significantly the total computational cost. In this section, we avoid these adjoint computations by decomposing the shape derivative of the eigenmodes on the (already computed) modal basis.

To simplify the presentation, we again focus on the single-frequency case

$$
J_{\omega}(\Omega)=J_{\omega}^{\bmod }\left(\left\{\omega_{j}, r_{j}\right\}_{j=1}^{\infty}\right) .
$$

Proposition 6.1. Assume that the damping is not zero and is modelled by (26), with $\xi_{j}>0$ for all modes. Assume that all eigenfrequencies $\omega_{j}$ are simple. The shape derivative of the single-frequency dynamic compliance is

$$
\begin{aligned}
J_{\omega}^{\prime}(\Omega)(\theta)= & \sum_{j=1}^{\infty}\left(\frac{d^{j, j}}{2 \omega_{j}} \int_{\Gamma}\left(A e\left(r_{j}\right) \cdot e\left(r_{j}\right)-\omega_{j}^{2} \rho\left|r_{j}\right|^{2}\right) \theta \cdot n d s-\frac{d_{\mathrm{Re}}^{j, j}+d_{\mathrm{Im}}^{j, j}}{2} \int_{\Gamma} \rho\left|r_{j}\right|^{2} \theta \cdot n d s\right. \\
& \left.+\sum_{i=1, i \neq j}^{\infty} \frac{d_{\mathrm{Re}}^{j, i}+d_{\mathrm{Im}}^{j, i}}{\left(\omega_{i}^{2}-\omega_{j}^{2}\right)} \int_{\Gamma}\left(\omega_{j}^{2} \rho r_{j} \cdot r_{i}-A e\left(r_{j}\right) \cdot e\left(r_{i}\right)\right) \theta \cdot n d s\right),
\end{aligned}
$$

where the various coefficients are defined in (63), (64) and (65).

Remark 6.1. Formula (45) satisfies the Hadamard structure theorem (i.e., depends only on the normal component of the vector field $\theta$ on the boundary $\Gamma$ ) and thus a descent direction is readily revealed. A similar formula would hold for any other objective function, different from the dynamic compliance, of course with different values of the coefficients. Note that, in Proposition 6.1, no adjoint state is required, independently of the objective function that is considered. The reason is that instead of using an adjoint state to avoid the computation of the shape derivative of the state variable, we shall decompose it into the modal basis. The counterpart is the presence of a double summation in (45). In numerical practice, formula (45) will be approximated with a finite number $n_{\text {mod }}$ of eigenmodes. 
Remark 6.2. The shape derivative of the dynamic compliance (10) is recovered from (45) by integrating with respect to $\omega$, namely

$$
\begin{gathered}
J^{\prime}(\Omega)(\theta)=\int_{\omega_{\min }}^{\omega_{\max }} J_{\omega}^{\prime}(\Omega)(\theta) d \omega=\sum_{j=1}^{\infty}\left(\frac{\widetilde{d}^{j, j}}{2 \omega_{j}} \int_{\Gamma}\left(A e\left(r_{j}\right) \cdot e\left(r_{j}\right)-\omega_{j}^{2} \rho\left|r_{j}\right|^{2}\right) \theta \cdot n d s\right. \\
\left.-\frac{\widetilde{d}_{\mathrm{Re}}^{j, j}+\widetilde{d}_{\mathrm{Im}}^{j, j}}{2} \int_{\Gamma} \rho\left|r_{j}\right|^{2} \theta \cdot n d s+\sum_{i=1, i \neq j}^{\infty} \frac{\widetilde{d}_{\mathrm{Re}}^{j, i}+\widetilde{d}_{\mathrm{Im}}^{j, i}}{\left(\omega_{i}^{2}-\omega_{j}^{2}\right)} \int_{\Gamma}\left(\omega_{j}^{2} \rho r_{j} \cdot r_{i}-A e\left(r_{j}\right) \cdot e\left(r_{i}\right)\right) \theta \cdot n d s\right),
\end{gathered}
$$

where

$$
\widetilde{d}_{\mathrm{Re}, \mathrm{Im}}^{i, j}=\int_{\omega_{\min }}^{\omega_{\max }} d_{\mathrm{Re}, \operatorname{Im}}^{i, j} d \omega .
$$

In (46) we rely on the fact that all integrals on $\Gamma$ do not depend on the excitation frequency $\omega$. This will be taken into account for evaluating the computational efficiency of this formula in Section 8.

Lemma 6.1. Assume that all eigenfrequencies $\omega_{j}$ are simple. For any vector field $\theta \in W^{1, \infty}\left(\mathbb{R}^{d}, \mathbb{R}^{d}\right)$, the Eulerian shape derivative $\Omega_{j}(\theta)$ of $\omega_{j}$ is given by

$$
\Omega_{j}(\theta)=\frac{1}{2 \omega_{j}} \int_{\Gamma}\left(A e\left(r_{j}\right) \cdot e\left(r_{j}\right)-\omega_{j}^{2} \rho\left|r_{j}\right|^{2}\right) \theta \cdot n d s .
$$

and the Eulerian shape derivatives $U_{j}(\theta)$ of $r_{j}$ is the unique solution of

$$
\left\{\begin{aligned}
-\operatorname{div}\left(A e\left(U_{j}(\theta)\right)\right)-\omega_{j}^{2} \rho U_{j}(\theta) & =2 \omega_{j} \Omega_{j}(\theta) \rho r_{j} & & \text { in } \Omega, \\
U_{j}(\theta) & =0 & & \text { on } \Gamma_{D}, \\
\left(A e\left(U_{j}(\theta)\right)\right) n & =0 & & \text { on } \Gamma_{N}, \\
\left(A e\left(U_{j}(\theta)\right)\right) n & =L_{j}(\theta) & & \text { on } \Gamma,
\end{aligned}\right.
$$

normalized by

$$
\int_{\Omega} 2 \rho r_{j} \cdot U_{j} d x+\int_{\Gamma} \rho\left|r_{j}\right|^{2} \theta \cdot n d s=0,
$$

where $L_{j}(\theta)$ is a surface load defined by (56). Decomposing $U_{j}(\theta)$ on the modal basis yields

$$
\begin{aligned}
& U_{j}=\sum_{i=1}^{\infty} a_{i}^{j} r_{i}, \text { with } a_{j}^{j}=-\frac{1}{2} \int_{\Gamma} \rho\left|r_{j}\right|^{2} \theta \cdot n d s \\
& \text { and, for } i \neq j, \quad a_{i}^{j}=\frac{1}{\left(\omega_{i}^{2}-\omega_{j}^{2}\right)} \int_{\Gamma}\left(\omega_{j}^{2} \rho r_{j} \cdot r_{i}-A e\left(r_{j}\right) \cdot e\left(r_{i}\right)\right) \theta \cdot n d s .
\end{aligned}
$$

Proof. Recall that the boundaries $\Gamma_{D}$ and $\Gamma_{N}$ are not optimized and kept fixed (in other words, we have $\theta=0$ on $\left.\Gamma_{D} \cup \Gamma_{N}\right)$. By the differentiability of simple eigenfunctions [17], we know that the shape derivatives $\Omega_{j}(\theta)$ and $U_{j}(\theta)$ exist and are uniquely defined. Recalling the definition (17) of the Hilbert space $V$, the variational formulation of $(22)$ is

$$
\int_{\Omega} A e\left(r_{j}\right) \cdot e(\phi) d x=\omega_{j}^{2} \int_{\Omega} \rho r_{j} \cdot \phi d x \quad \text { for any } \phi \in V .
$$

Taking the shape derivative of the previous equation yields

$$
\begin{aligned}
\int_{\Omega} A e\left(U_{j}(\theta)\right) \cdot e(\phi) d x+\int_{\Gamma} A e\left(r_{j}\right) \cdot e(\phi) \theta \cdot n d s= & 2 \omega_{j} \Omega_{j}(\theta) \int_{\Omega} \rho r_{j} \cdot \phi d x+\omega_{j}^{2} \int_{\Omega} \rho U_{j}(\theta) \cdot \phi d x \\
& +\omega_{j}^{2} \int_{\Gamma} \rho r_{j} \cdot \phi \theta \cdot n d s .
\end{aligned}
$$


Then choosing $\phi=r_{j}$ and recalling the normalization (23) of $r_{j}$, we deduce

$$
\int_{\Gamma} A e\left(r_{j}\right) \cdot e\left(r_{j}\right) \theta \cdot n d s=2 \omega_{j} \Omega_{j}(\theta)+\omega_{j}^{2} \int_{\Gamma} \rho r_{j} \cdot r_{j} \theta \cdot n d s,
$$

which is precisely (47). Choosing $\phi$ with compact support in $\Omega$, equation (52) leads to the first equation of (48)

$$
-\operatorname{div}\left(A e\left(U_{j}(\theta)\right)\right)-\omega_{j}^{2} \rho U_{j}(\theta)=2 \omega_{j} \Omega_{j}(\theta) \rho r_{j}, \quad \text { in } \Omega .
$$

Furthermore, since the boundary $\Gamma_{D}$ is not optimized and is fixed, the shape derivative $U_{j}(\theta)$ satisfies the same boundary condition $U_{j}(\theta)=0$ on $\Gamma_{D}$. Multiplying (53) by a test function $\phi \in V$, integrating by parts and substracting the result to (52) leads to

$$
\int_{\Gamma_{N} \cup \Gamma}\left(A e\left(U_{j}\right) \cdot n\right) \cdot \phi d s=-\int_{\Gamma} A e\left(r_{j}\right) \cdot e(\phi) \theta \cdot n d s+\omega_{j}^{2} \int_{\Gamma} \rho r_{j} \cdot \phi \theta \cdot n d s .
$$

Taking $\phi$ with compact support in $\Gamma_{N}$, we deduce that $U_{j}(\theta)$ satisifies a homogeneous Neumann boundary condition on $\Gamma_{N}$. On the other hand, taking $\phi$ with compact support in $\Gamma$, and using the Neumann boundary condition of $r_{j},(54)$ becomes

$$
\int_{\Gamma}\left(A e\left(U_{j}\right) \cdot n\right) \cdot \phi d s=-\int_{\Gamma}\left[A e\left(r_{j}\right)\right]_{t} \cdot[e(\phi)]_{t} \theta \cdot n d s+\omega_{j}^{2} \int_{\Gamma} \rho r_{j} \cdot \phi \theta \cdot n d s,
$$

where the notation $[M]_{t}$ denotes the $(d-1)$-dimensional projection of the matrix $M$ on the tangent space to $\Gamma$. Then, using some tangential integration by parts (see [14] for details), one can check that (55) defines a surface load $L_{j}(\theta)$ as

$$
\int_{\Gamma} L_{j}(\theta) \cdot \phi d s=-\int_{\Gamma}\left[A e\left(r_{j}\right)\right]_{t} \cdot[e(\phi)]_{t} \theta \cdot n d s+\omega_{j}^{2} \int_{\Gamma} \rho r_{j} \cdot \phi \theta \cdot n d s,
$$

which yields a non-homogeneous Neumann boundary condition for $U_{j}(\theta)$ on $\Gamma$.

We now decompose $U_{j}$ in the modal basis, and our goal is to compute its coefficients $a_{i}^{j}$. Taking $\phi=r_{i}, i \neq j$ in (52), by orthogonality of the eigenfunctions, we get:

$$
\int_{\Omega}\left(A e\left(U_{j}\right) \cdot e\left(r_{i}\right)-\omega_{j}^{2} \rho U_{j} \cdot r_{i}\right) d x=-\int_{\Gamma} A e\left(r_{j}\right) \cdot e\left(r_{i}\right)(\theta \cdot n) d s+\omega_{j}^{2} \int_{\Gamma} \rho r_{j} \cdot r_{i}(\theta \cdot n) d s .
$$

Taking $\phi=U_{j}$ in (51), we obtain:

$$
\int_{\Omega} A e\left(r_{i}\right) \cdot e\left(U_{j}\right) d x=\omega_{i}^{2} \int_{\Omega} \rho r_{i} \cdot U_{j} d x .
$$

Combining (58) and (57), we get:

$$
\left(\omega_{i}^{2}-\omega_{j}^{2}\right) \int_{\Omega} \rho U_{j} \cdot r_{i} d x=-\int_{\Gamma} A e\left(r_{j}\right) \cdot e\left(r_{i}\right)(\theta \cdot n) d s+\omega_{j}^{2} \int_{\Gamma} \rho r_{j} \cdot r_{i}(\theta \cdot n) d s
$$

and thus, for $i \neq j$, we obtain the value of the coefficient $a_{i}^{j}$ in (50). For $i=j$, differentiating the normalization condition (23) for $r_{j}$ yields

$$
\int_{\Omega} 2 \rho r_{j} \cdot U_{j} d x+\int_{\Gamma} \rho r_{j} \cdot r_{j} \theta \cdot n d s=0
$$

from which we deduce by orthogonality the value of the coefficient $a_{j}^{j}$ in (50). 
Proof of Proposition 6.1. The shape derivative of the single-frequency objective function is

$$
J_{\omega}^{\prime}(\Omega)(\theta)=\left\langle\frac{\partial J_{\omega}}{\partial u_{\mathrm{Re}}}, u_{\mathrm{Re}}^{\prime}(\theta)\right\rangle+\left\langle\frac{\partial J_{\omega}}{\partial u_{\mathrm{Im}}}, u_{\mathrm{Im}}^{\prime}(\theta)\right\rangle
$$

which yields

$$
J_{\omega}^{\prime}(\Omega)(\theta)=\sum_{j=1}^{\infty}\left(\left\langle\frac{\partial J_{\omega}}{\partial u_{\mathrm{Re}}},\left(\frac{\partial u_{\mathrm{Re}}}{\partial \omega_{j}} \Omega_{j}(\theta)+\frac{\partial u_{\mathrm{Re}}}{\partial r_{j}} U_{j}(\theta)\right)\right\rangle+\left\langle\frac{\partial J_{\omega}}{\partial u_{\mathrm{Im}}},\left(\frac{\partial u_{\mathrm{Im}}}{\partial \omega_{j}} \Omega_{j}(\theta)+\frac{\partial u_{\mathrm{Im}}}{\partial r_{j}} U_{j}(\theta)\right)\right\rangle\right),
$$

where $\Omega_{j}(\theta)$ and $U_{j}(\theta)$ are the shape derivatives of $\omega_{j}$ and $r_{j}$ respectively, given in Lemma 6.1 . We split (60) in several parts to simplify the presentation:

$$
J_{\omega}^{\prime}(\Omega)(\theta)=\sum_{j=1}^{\infty}\left(D_{\mathrm{Re}, \omega}^{j}+D_{\mathrm{Re}, r}^{j}+D_{\mathrm{Im}, \omega}^{j}+D_{\mathrm{Im}, r}^{j}\right)
$$

where

$$
\begin{array}{ll}
D_{\mathrm{Re}, \omega}^{j}=\Omega_{j}(\theta)\left\langle\frac{\partial J_{\omega}}{\partial u_{\mathrm{Re}}}, \frac{\partial u_{\mathrm{Re}}}{\partial \omega_{j}}\right\rangle, & D_{\mathrm{Re}, r}^{j}=\left\langle\frac{\partial J_{\omega}}{\partial u_{\mathrm{Re}}}, \frac{\partial u_{\mathrm{Re}}}{\partial r_{j}} U_{j}(\theta)\right\rangle, \\
D_{\operatorname{Im}, \omega}^{j}=\Omega_{j}(\theta)\left\langle\frac{\partial J_{\omega}}{\partial u_{\mathrm{Im}}}, \frac{\partial u_{\mathrm{Im}}}{\partial \omega_{j}}\right\rangle, & D_{\mathrm{Im}, r}^{j}=\left\langle\frac{\partial J_{\omega}}{\partial u_{\mathrm{Im}}}, \frac{\partial u_{\mathrm{Im}}}{\partial r_{j}} U_{j}(\theta)\right\rangle .
\end{array}
$$

First, note that the directional derivatives of the dynamic compliance $J_{\omega}$, for any $\phi \in V$, are given by

$$
\left\langle\frac{\partial J_{\omega}}{\partial u_{\mathrm{Re}}}, \phi\right\rangle=\int_{\Gamma_{N}} f_{\mathrm{Im}} \cdot \phi d s \quad \text { and } \quad\left\langle\frac{\partial J_{\omega}}{\partial u_{\mathrm{Im}}}, \phi\right\rangle=-\int_{\Gamma_{N}} f_{\mathrm{Re}} \cdot \phi d s .
$$

Recall from (40) that

$$
\frac{\partial u_{\mathrm{Re}}}{\partial \omega_{j}}=\beta_{\operatorname{Re}}\left(\omega_{j}, r_{j}\right) r_{j} \quad \text { and } \quad \frac{\partial u_{\operatorname{Im}}}{\partial \omega_{j}}=\beta_{\operatorname{Im}}\left(\omega_{j}, r_{j}\right) r_{j} .
$$

Therefore

$$
D_{\mathrm{Re}, \omega}^{j}=\Omega_{j}(\theta) \int_{\Gamma_{N}} \beta_{\operatorname{Re}}\left(\omega_{j}, r_{j}\right) f_{\operatorname{Im}} \cdot r_{j} d s \quad \text { and } \quad D_{\operatorname{Im}, \omega}^{j}=-\Omega_{j}(\theta) \int_{\Gamma_{N}} \beta_{\operatorname{Im}}\left(\omega_{j}, r_{j}\right) f_{\operatorname{Re}} \cdot r_{j} d s
$$

and thus

$$
D_{\mathrm{Re}, \omega}^{j}=\beta_{\mathrm{Re}}\left(\omega_{j}, r_{j}\right)\left(\int_{\Gamma_{N}} f_{\mathrm{Im}} \cdot r_{j} d s\right)\left(\int_{\Gamma} \frac{1}{2 \omega_{j}}\left(A e\left(r_{j}\right) \cdot e\left(r_{j}\right)-\omega_{j}^{2} \rho\left|r_{j}\right|^{2}\right) \theta \cdot n d s\right)
$$

and

$$
D_{\mathrm{Im}, \omega}^{j}=-\beta_{\operatorname{Im}}\left(\omega_{j}, r_{j}\right)\left(\int_{\Gamma_{N}} f_{\mathrm{Re}} \cdot r_{j} d s\right)\left(\int_{\Gamma} \frac{1}{2 \omega_{j}}\left(A e\left(r_{j}\right) \cdot e\left(r_{j}\right)-\omega_{j}^{2} \rho\left|r_{j}\right|^{2}\right) \theta \cdot n d s\right) .
$$

It simplifies as

$$
D_{\mathrm{Re}, \omega}^{j}+D_{\mathrm{Im}, \omega}^{j}=\frac{d^{j, j}}{2 \omega_{j}} \int_{\Gamma}\left(A e\left(r_{j}\right) \cdot e\left(r_{j}\right)-\omega_{j}^{2} \rho\left|r_{j}\right|^{2}\right) \theta \cdot n d s
$$

with

$$
d^{j, j}=\beta_{\mathrm{Re}}\left(\omega_{j}, r_{i}\right)<f_{\operatorname{Im}}, r_{j}>_{\Gamma_{N}}-\beta_{\operatorname{Im}}\left(\omega_{j}, r_{j}\right)<f_{\mathrm{Re}}, r_{j}>_{\Gamma_{N}} .
$$


On the other hand, from (36) we deduce

$$
\left\langle\frac{\partial u_{\mathrm{Re}}}{\partial r_{j}}, U_{j}(\theta)\right\rangle=\alpha_{\operatorname{Re}}\left(\omega_{j}, r_{j}\right) U_{j}(\theta)+\alpha_{\operatorname{Re}}\left(\omega_{j}, U_{j}(\theta)\right) r_{j}
$$

and replacing $U_{j}(\theta)$ by its modal decomposition we obtain

$$
\left\langle\frac{\partial u_{\mathrm{Re}}}{\partial r_{j}}, U_{j}(\theta)\right\rangle=\sum_{i=1}^{\infty} a_{i}^{j}\left(\alpha_{\operatorname{Re}}\left(\omega_{j}, r_{j}\right) r_{i}+\alpha_{\operatorname{Re}}\left(\omega_{j}, r_{i}\right) r_{j}\right) .
$$

Then, from (62) we get

$$
D_{\mathrm{Re}, r}^{j}=\int_{\Gamma_{N}} f_{\operatorname{Im}} \cdot\left\langle\frac{\partial u_{\mathrm{Re}}}{\partial r_{j}}, U_{j}(\theta)\right\rangle d s=\sum_{i=1}^{\infty} a_{i}^{j}\left(\alpha_{\mathrm{Re}}\left(\omega_{j}, r_{j}\right) \int_{\Gamma_{N}} f_{\operatorname{Im}} \cdot r_{i} d s+\alpha_{\operatorname{Re}}\left(\omega_{j}, r_{i}\right) \int_{\Gamma_{N}} f_{\operatorname{Im}} \cdot r_{j} d s\right)
$$

Finally, $D_{\mathrm{Re}, r}^{j}$ reads:

$$
D_{\mathrm{Re}, r}^{j}=\int_{\Gamma}(\theta \cdot n)\left[\sum_{i=1, i \neq j}^{\infty} \frac{1}{\left(\omega_{i}^{2}-\omega_{j}^{2}\right)}\left(\omega_{j}^{2} \rho r_{j} \cdot r_{i}-A e\left(r_{j}\right) \cdot e\left(r_{i}\right)\right) d_{\mathrm{Re}}^{j, i}-\left(\frac{1}{2} \rho\left|r_{j}\right|^{2}\right) d_{\mathrm{Re}}^{j, j}\right] d s,
$$

with

$$
d_{\mathrm{Re}}^{j, i}=\alpha_{\operatorname{Re}}\left(\omega_{j}, r_{i}\right)<f_{\operatorname{Im}}, r_{j}>_{\Gamma_{N}}+\alpha_{\operatorname{Re}}\left(\omega_{j}, r_{j}\right)<f_{\operatorname{Im}}, r_{i}>_{\Gamma_{N}} .
$$

A similar computation holds for $D_{\mathrm{Im}, r}^{j}$ which is given by

$$
D_{\operatorname{Im}, r}^{j}=\int_{\Gamma}(\theta \cdot n)\left[\sum_{i=1, i \neq j}^{\infty} \frac{1}{\left(\omega_{i}^{2}-\omega_{j}^{2}\right)}\left(\omega_{j}^{2} \rho r_{j} \cdot r_{i}-A e\left(r_{j}\right) \cdot e\left(r_{i}\right)\right) d_{\operatorname{Im}}^{j, i}-\left(\frac{1}{2} \rho\left|r_{j}\right|^{2}\right) d_{\mathrm{Im}}^{j, j}\right] d s,
$$

with

$$
d_{\operatorname{Im}}^{j, i}=-\alpha_{\operatorname{Im}}\left(\omega_{j}, r_{i}\right)<f_{\mathrm{Re}}, r_{j}>_{\Gamma_{N}}-\alpha_{\operatorname{Im}}\left(\omega_{j}, r_{j}\right)<f_{\mathrm{Re}}, r_{i}>_{\Gamma_{N}} .
$$

Collecting all these terms leads to (45).

Remark 6.3. There is an alternative proof of Proposition 6.1 which starts from the result of Proposition 5.1 and decomposes the adjoint state $q_{j}$ on the modal basis. We checked, with some tedious algebra, that it leads to the same formula (45).

Remark 6.4. Note that our analysis is mathematically rigorous if all eigenvalues are assumed to be simple, of multiplicity equal to one. However, the final result (45) does not explicitly depend on the multiplicity of eigenvalues. Actually, we expect it could be generalized by using the differentiability of the eigen-projectors when multiple eigenvalues appear. In any case, from a numerical point of view, we shall use it without checking the simplicity of eigenvalues.

Remark 6.5. In the proof of Proposition 6.1 it is crucial to assume that the damping operator $C$ is diagonal in the modal basis. Otherwise, the decomposition of the state $u_{\mathrm{Re}}, u_{\mathrm{Im}}$ in the modal basis would require to solve a linear system. However, such an assumption on the diagonal character of the damping is common practice in frequency response problems.

Remark 6.6. In the proof of Proposition 6.1, it was possible to decompose the eigenvector's derivative $U_{j}(\theta)$ onto the modal basis $\left\{r_{k}\right\}_{k=1, \ldots, \infty}$ because they all satisfy the same homogeneous Dirichlet boundary condition on $\Gamma_{D}$. This is possible since $\Gamma_{D}$ is not optimized (we have set $\theta=0$ on $\Gamma_{D}$ ), which results in homogeneous Dirichlet conditions for $U_{j}$ on $\Gamma_{D}$. When $\Gamma_{D}$ is subject to optimization and can move, then $U_{j}(\theta)$ satisfies a non-homogeneous Dirichlet boundary condition on $\Gamma_{D}$ [1], [14], [30], [31]. Therefore, it is not possible to decompose $U_{j}(\theta)$ onto the modal basis and one should rather decompose the Lagrangian shape derivative of the eigenvector $r_{j}$, given by the relation $V_{j}=U_{j}+\theta \cdot \nabla r_{j}$, which satisfies $V_{j}=0$ on $\Gamma_{D}$. 


\section{Complete modal decomposition of the state and adjoint problems}

Eventually we discuss a last approach which turns out to be the simplest one and yields a formula for the shape derivative which is similar, albeit different, from that of Proposition 6.1. Starting from the standard approach of Section 4, the main idea is to decompose both solutions of the direct and adjoint problems in the modal basis of Section 5.

We first rephrase Proposition 4.1 in the case where the multiplicative damping coefficient $c(x)$ is replaced by a modal damping operator $C$, defined by (26). In other words, compared to Section 4 , the state equation (4) is replaced by (24). As before, the objective function is the single-frequency dynamic compliance

$$
J_{\omega}(\Omega)=\int_{\Gamma_{N}}\left(f_{\mathrm{Im}} \cdot u_{\operatorname{Re}}(\omega)-f_{\operatorname{Re}} \cdot u_{\operatorname{Im}}(\omega)\right) d s .
$$

Corollary 7.1. Assume that the damping is an operator $C$, defined by (26). The shape derivative of the single-frequency dynamic compliance is

$$
\begin{gathered}
J_{\omega}^{\prime}(\Omega)(\theta)=\int_{\Gamma}\left(-\omega^{2} \rho\left(u_{\mathrm{Re}} \cdot p_{\operatorname{Re}}+u_{\operatorname{Im}} \cdot p_{\mathrm{Im}}\right)-\omega\left(C\left(u_{\mathrm{Im}}\right) \cdot p_{\mathrm{Re}}-C\left(u_{\mathrm{Re}}\right) \cdot p_{\mathrm{Im}}\right)\right. \\
\left.+A e\left(u_{\mathrm{Re}}\right) \cdot e\left(p_{\mathrm{Re}}\right)+A e\left(u_{\mathrm{Im}}\right) \cdot e\left(p_{\mathrm{Im}}\right)\right) \theta \cdot n d s,
\end{gathered}
$$

where $P=p_{\operatorname{Re}}+i p_{\operatorname{Im}}$ is the adjoint state, solution of

$$
\left\{\begin{aligned}
-\omega^{2} \rho P-i \omega C(P)-\operatorname{div}(A e(P)) & =0 & & \text { in } \Omega, \\
P & =0 & & \text { on } \Gamma_{D}, \\
(A e(P)) n & =i F & & \text { on } \Gamma_{N}, \\
(A e(P)) n & =0 & & \text { on } \Gamma .
\end{aligned}\right.
$$

The proof of Corollary 7.1 is identical to that of Proposition 4.1, once it is recognized that $C$, defined by $(26)$, is a self-adjoint operator. It remains to decompose $u_{\mathrm{Re}}, u_{\mathrm{Im}}, p_{\mathrm{Re}}, p_{\mathrm{Im}}$ in the modal basis to obtain our new result.

Proposition 7.1. Assume that the damping is an operator $C$, defined by (26). The shape derivative of the single-frequency dynamic compliance is given by (66) where the state, solution of (24), is decomposed as

$$
U(x)=u_{\mathrm{Re}}+i u_{\mathrm{Im}}=\sum_{j=1}^{\infty}\left(a_{\mathrm{Re}}^{j}+i a_{\mathrm{Im}}^{j}\right) r_{j}(x),
$$

with $a_{\mathrm{Re}}^{j}, a_{\mathrm{Im}}^{j}$, defined by (25) and (27), and the adjoint, solution of (67), is decomposed as

$$
P(x)=p_{\operatorname{Re}}+i p_{\operatorname{Im}}=\sum_{j=1}^{\infty}\left(b_{\mathrm{Re}}^{j}+i b_{\mathrm{Im}}^{j}\right) r_{j}(x),
$$

with

$$
\begin{aligned}
b_{\mathrm{Re}}^{j} & =-\frac{<f_{\mathrm{Im}}, r_{j}>_{\Gamma_{N}}\left(\omega_{j}^{2}-\omega^{2}\right)+<f_{\mathrm{Re}}, r_{j}>_{\Gamma_{N}} 2 \omega \omega_{j} \xi_{j}}{\left(\omega_{j}^{2}-\omega^{2}\right)^{2}+\left(2 \omega \omega_{j} \xi_{j}\right)^{2}}, \\
b_{\mathrm{Im}}^{j} & =\frac{<f_{\mathrm{Re}}, r_{j}>_{\Gamma_{N}}\left(\omega_{j}^{2}-\omega^{2}\right)-<f_{\mathrm{Im}}, r_{j}>_{\Gamma_{N}} 2 \omega \omega_{j} \xi_{j}}{\left(\omega_{j}^{2}-\omega^{2}\right)^{2}+\left(2 \omega \omega_{j} \xi_{j}\right)^{2}} .
\end{aligned}
$$


Proof. In Section 5, we proved that $u_{\mathrm{Re}}, u_{\mathrm{Im}}$ are decomposed in the modal basis according to equations (28) and (29) respectively. Following the same type of analysis, we decompose the adjoint state $P$, solution of (67), as in (69).

Remark 7.1. Formulas (45) and (66) for the shape derivative of the dynamic compliance should be equivalent although it is not easy to check. Here again, in the proof of Proposition 7.1 it is crucial to assume that the damping operator $C$ is diagonal in the modal basis. Of course, the approach of Proposition 7.1 can be applied to any other objective function, different from the dynamic compliance: the definition of the adjoint equation will simply change.

Remark 7.2. One advantage of this method is that one does not need to assume the differentiability of the eigenvalues and eigenvectors at play. In fact, since the modal basis is used only for the approximation of the formulas derived via a frequency-domain analysis, the eigenvalues and eigenvectors are not differentiated. Thus, even in case of multiplicity greater than one, one shall not expect any problem to occur, assuming always that the number of modes considered is sufficiently high to guarantee an accurate enough approximation.

\section{Complexity of the different approaches}

This section is devoted to a brief comparison of the four optimization strategies presented in the four previous sections. Our comparison is made in terms of complexity or rough operation count.

The main parameters in this comparison are the three following numbers: the number $n_{\text {dof }}$ of degrees of freedom in the finite element analysis, the number $n_{\omega}$ of frequencies which are used to discretize the interval $\left[\omega_{\min }, \omega_{\max }\right]$, and the number $n_{\bmod }$ of eigenmodes in the modal basis. A finite element analysis amounts to solve a linear system of size $n_{\text {dof }}$. It can be performed by a direct method or by an iterative one like the conjugate gradient method. In any case we call $S\left(n_{\text {dof }}\right)$ the cost of such a linear solver in terms of floating point operations. This cost $S\left(n_{\text {dof }}\right)$ scales like $\mathcal{O}\left(n_{\text {dof }}^{q}\right)$ with $1<q \leq 3$. The value of the exponent $q$ varies with the type of method and the storage of the rigidity matrix.

The next ingredient is the computation of a truncated modal basis with $n_{\text {mod }}$ modes. Note that all previous formulas which involve a summation over all modes are approximated in numerical practice by a finite sum over the $n_{\text {mod }}$ first modes of the modal basis. Computing $n_{\text {mod }}$ eigenfrequencies and eigenmodes has cost which we denote by $E\left(n_{\bmod }, n_{\text {dof }}\right)$ and is typically larger than $S\left(n_{\text {dof }}\right)$ but smaller than $n_{\text {mod }} S\left(n_{\text {dof }}\right)$. Its precise value is not explicit since all algorithms for computing eigenvalues and eigenmodes are iterative.

In all our numerical applications and in the following discussion, the number of modes $n_{\bmod }$ is much smaller than the number of discrete excitation frequencies $n_{\omega}$ and the number of degrees of freedom $n_{\text {dof }}$. Typical values are $n_{\text {mod }} \approx 50, n_{\omega} \approx 500$ and $n_{\text {dof }}$ ranges from $10^{4}$ to $10^{6}$.

The direct approach of Section 4 is certainly the most costly one of all four. It requires to solve $n_{\omega}$ times the direct and adjoint systems. Even if the rigidity matrix is factorized, which implies that solving for the adjoint is at almost no extra cost after solving for the direct state, the rigidity matrices are different for all frequencies. Therefore, the overall cost is at least of the order of $\mathcal{O}\left(n_{\omega} S\left(n_{\text {dof }}\right)\right)$, which is unaffordable for most realistic cases

$$
\operatorname{Cost}(\text { Section } 4)=\mathcal{O}\left(n_{\omega} S\left(n_{\text {dof }}\right)\right)
$$

The approach of Section 5, based on a modal decomposition and an adjoint analysis for each mode, is the most classical one. Its cost is much more reasonable since it is

$$
\operatorname{Cost}(\operatorname{Section} 5)=E\left(n_{\text {mod }}, n_{\text {dof }}\right)+\mathcal{O}\left(n_{\text {mod }} S\left(n_{\text {dof }}\right)\right)+\mathcal{O}\left(n_{\omega} n_{\text {dof }}+n_{\text {mod }} n_{\text {dof }}\right),
$$


corresponding to first computing the modal basis, then solving the adjoint equation for each eigenmode in the modal basis after having averaged their right hand sides with respect to $\omega$ (see Remark 5.5), which is responsible for the last term $\mathcal{O}\left(n_{\omega} n_{\text {dof }}+n_{\text {mod }} n_{\text {dof }}\right)$. Indeed, computing these right hand sides involves as many integrals on $\Gamma_{N}$ as there are eigenmodes, which yields a cost of $\mathcal{O}\left(n_{\text {mod }} n_{\text {dof }}\right)$, and eventually averaging in $\omega$ yields a further cost of $\mathcal{O}\left(n_{\omega} n_{\text {dof }}\right)$. Actually, the number $n_{\text {dof }}$ is pessimistic in this operation count since integrals are computed on parts of the boundary and not on the full domain (but this is just a slight improvement). The operation count is much smaller for Section 5 than for Section 4 because $n_{\bmod } \ll n_{\omega}$ and the overhead cost of computing the modal basis does not override the gain in number of adjoints.

Our first new approach of Section 6 is based again on the modal decomposition but computes the eigenvectors' shape derivatives instead of using adjoints. Its cost is much more moderate

$$
\operatorname{Cost}(\text { Section } 6)=E\left(n_{\text {mod }}, n_{\text {dof }}\right)+\mathcal{O}\left(n_{\text {mod }}^{2} n_{\text {dof }}+n_{\text {mod }}^{2} n_{\omega}\right) \text {. }
$$

Indeed, formula (46) involves a double summation, namely $n_{\bmod }^{2}$ terms, each of them depends on the same integrals on $\Gamma_{N}$, which are $<f_{\mathrm{Re}}, r_{j}>_{\Gamma_{N}}$ and $<f_{\mathrm{Im}}, r_{i}>_{\Gamma_{N}}$ (see formulas (63), (64), (65), definitions (34), (35) of the coefficients $\alpha_{\mathrm{Re}, \mathrm{Im}}$ and definition (40) of the coefficients $\left.\beta_{\mathrm{Re}, \mathrm{Im}}\right)$. Computing these integrals requires of the order of $\mathcal{O}\left(n_{\bmod } n_{\mathrm{dof}}\right)$ floating point operations. Of course, these coefficients depend also on the excitation frequency $\omega$ and these computations are repeated for each of them, which yields the term $\left.\mathcal{O}\left(n_{\omega} n_{\bmod }^{2}\right)\right)$. Finally, formula (45) depends on combinations of the eigenmodes, independent of the excitation frequency, and their evaluation has a cost $\mathcal{O}\left(n_{\text {mod }}^{2} n_{\text {dof }}\right)$. In any case, the operation count of Section 6 outperforms that of Section 5 because no adjoint equations are solved and $n_{\text {mod }} \ll n_{\text {dof }}$.

Our second new approach of Section 7 has a (slightly) different operation count, compared to that of Section 6 .

$$
\operatorname{Cost}(\text { Section } 7)=E\left(n_{\text {mod }}, n_{\text {dof }}\right)+\mathcal{O}\left(n_{\omega} n_{\text {mod }} n_{\text {dof }}\right) .
$$

It is based on applying the modal decomposition to the states and adjoints of the first approach of Section 4. Therefore, one has first to compute the modal basis and then evaluate the various coefficients in formula (68) and (69). They all depend on the same integrals $<f_{\operatorname{Re}}, r_{j}>_{\Gamma_{N}}$ and $<f_{\mathrm{Im}}, r_{i}>_{\Gamma_{N}}$, which has a $\operatorname{cost} \mathcal{O}\left(n_{\mathrm{mod}} n_{\mathrm{dof}}\right)$. Then, the other operations are simply done for each eigenmode, each excitation frequency and at each point of $\Gamma$, which yields the $\left.\operatorname{cost} \mathcal{O}\left(n_{\omega} n_{\bmod } n_{\text {dof }}\right)\right)$. There is no double sum as in the previous approach of Section 6 , but one could first develop the state and adjoint in formula (66) along the modal basis and then come back to the operation count of Section 6. The difference is quite negligible in front of the cost of computing the modal basis.

As a conclusion, we claim that our two new approaches of Sections 6 and 7 outperform all other strategies, as far as the number $n_{\text {mod }}$ of modes in the modal basis is much smaller than the number $n_{\omega}$ of discrete excitation frequencies. Of course, it requires in the first place an efficient algorithm for computing the modal basis.

Remark 8.1. In all our numerical experiments we rely on the Scilab software [7] to compute the modal basis, which in turn is calling the ARPACK package [18]. The "eigs" routine of Scilab computes eigenvalues and eigenvectors by an Arnoldi algorithm. To give an idea of the required computing time for this Arnoldi algorithm, we consider the structure of Figure 3 on a $300 \times 100$ square mesh. On a standard laptop (Intel(R) Core(TM) i\%-4600U 2.70 GHz with 16GB RAM) it takes 18 seconds to compute the 10 first modes, 25 seconds for the 20 first ones, 62 seconds for the 40 first ones and 164 seconds for the 80 first modes.

\section{Numerical validation of the modal basis approach}

Before proceeding with numerical tests on the proposed non-adjoint methods, we first check that our choices of parameters in the modal analysis provide accurate enough results. The first param- 
eter to validate is the number of eigenmodes considered in the modal decomposition. The second one is the number of steps for discretizing the excitation frequency interval.

We perform our comparisons on an optimized shape for a frequency response problem. As a test case, we chose the classical two-dimensional MBB beam of dimensions $6 \mathrm{x} 1$. The vertical displacement is fixed at its lower-left point and the structure is clamped at its lower-right point (see Figure 1). A unit point load $g=(0,1)$ is applied at the middle of its upper side. Due to symmetry, only one half of the structure is discretized by 300x100 Q1 elements. The Young modulus and the density of the elastic material are normalized to 1 , while the complementary of the shape $(D \backslash \bar{\Omega})$ is occupied by an "ersatz" material with Young modulus $E=10^{-3}$ and density $\rho=10^{-5}$, while both materials have Poisson ratio 0.33.

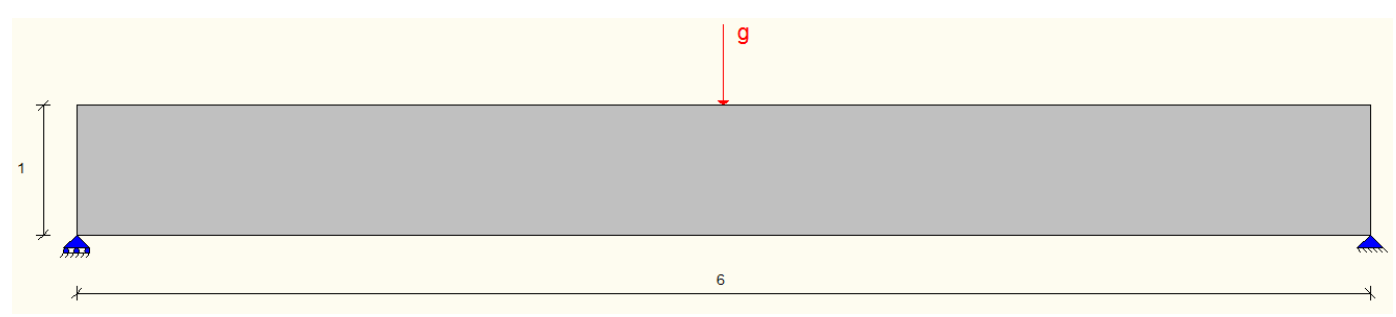

Figure 1: Boundary conditions for the 2d MBB beam.

To obtain meaningfull values of the constraints for the frequency response problem, we fist solve a simpler static optimization problem (without any vibrating force):

$$
\begin{array}{rl}
\min _{\Omega \in \mathcal{U}_{a d}} & V(\Omega) \\
\text { s.t. } & C(\Omega)=\int_{\Gamma_{N}} g \cdot u d s \leq C_{\max },
\end{array}
$$

where $u$ is the solution of

$$
\left\{\begin{aligned}
-\operatorname{div}(A e(u)) & =0 & & \text { in } \Omega, \\
u & =0 & & \text { on } \Gamma_{D}, \\
(A e(u)) n & =g & & \text { on } \Gamma_{N}, \\
(A e(u)) n & = & & \text { on } \Gamma .
\end{aligned}\right.
$$

The initialization as well as the optimized shape for $C_{\max }=150$ are shown in Figure 2 . The optimized shape, denoted by $\Omega^{r e f}$, will serve as a reference shape in the sequel. After 22 iterations, the total volume reduces from 2.013 to 0.415 , while the compliance for the optimal shape reads 149.99, starting from 87.51 for the initial shape. 


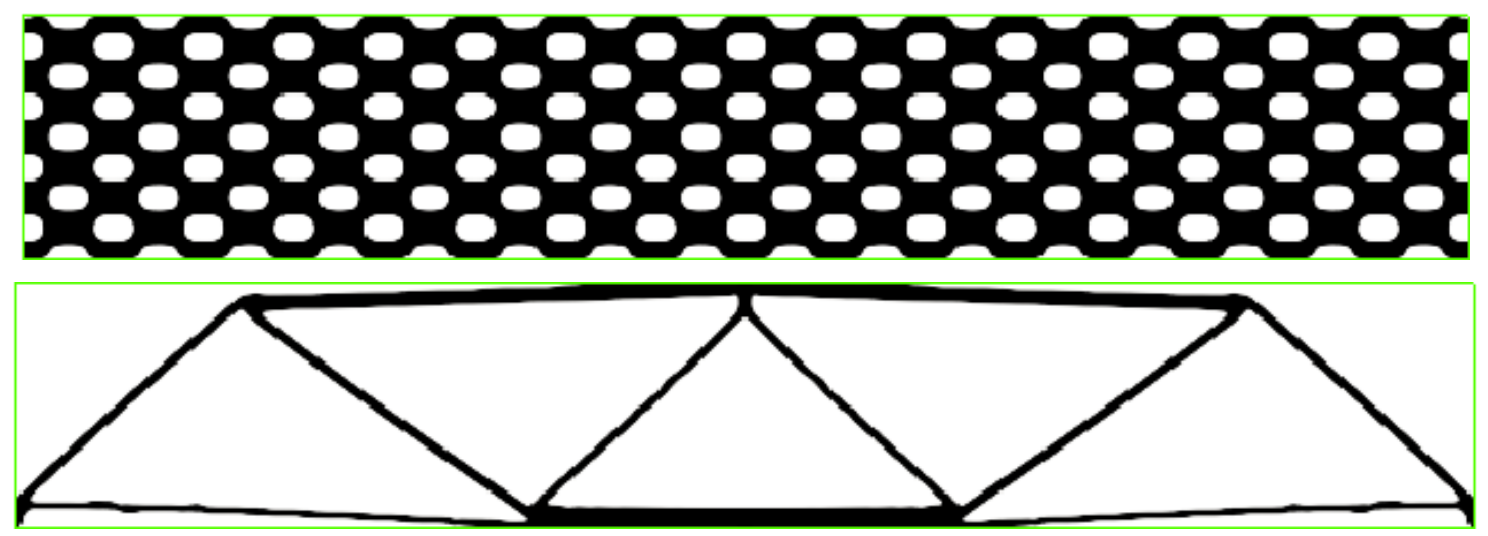

Figure 2: Up: initialization; down: optimized shape $\Omega^{\text {ref }}$ for the static compliance problem (72).

Now, we turn to the definition of the frequency response problem. We add a constraint on the dynamic compliance of the structure to account for vibrations of the external force of the type $\cos (\omega t)(0,1)$ in the frequency interval $\left[\omega_{\min }, \omega_{\max }\right]$ with $\omega_{\min }=0.02 \mathrm{~Hz}$ and $\omega_{\max }=0.1 \mathrm{~Hz}$. This interval is chosen so that it includes several of the first eigenvalues of the optimized shape of Figure 2 .

In order to make fair comparisons between the results obtained via the modal decomposition and the ones obtained via a classical finite element analysis, presented in Section 4, we have to use the same modelling for the damping. Since in the classical approach the damping cannot be specified mode by mode, we model the damping matrix as $C=2 \xi M$ with $\xi=0.05$ and $M$ the mass matrix. In other words, the damping coefficients, defined in (26), are set equal to $C_{k k}=2 \xi M_{k k}=2 \xi$, since $M_{k k}=1$ by normalization. Recalling the definition of the dynamic compliance

$$
C_{d y n}(\Omega)=\int_{\omega_{\min }}^{\omega_{\max }} \int_{\Gamma_{N}}\left(g_{\operatorname{Im}} \cdot u_{\operatorname{Re}}(\omega)-g_{\operatorname{Re}} \cdot u_{\operatorname{Im}}(\omega)\right) d s d \omega,
$$

with $g_{\operatorname{Re}}=g=(0,1), g_{\mathrm{Im}}=0$ and $\left(u_{\operatorname{Re}}, u_{\mathrm{Im}}\right)$ solution of the direct problem (5) and (6) (with loads $g$ rather than $f$ ), the new optimization problem reads:

$$
\begin{array}{rl}
\min _{\Omega \in \mathcal{U}_{a d}} & V(\Omega) \\
\text { s.t. } & C(\Omega)=\int_{\Gamma_{N}} g \cdot u d s \leq C_{\max } \\
& C_{d y n}(\Omega) \leq \alpha C_{d y n}\left(\Omega^{r e f}\right), \alpha \in(0,1),
\end{array}
$$

where $C_{d y n}\left(\Omega^{r e f}\right)=14.82$ stands for the dynamic compliance of the reference shape $\Omega^{\text {ref }}$ that solves problem (72) (lower shape in Figure 2).

Solving the optimization problem (73) for $\alpha=0.3$, starting from the same initialization as for problem (72) (upper shape in Figure 2) and using the method described in Section 7, we obtain after 56 iterations the optimized shape of Figure 3, whose dynamic compliance is $C_{d y n}\left(\Omega^{o p t}\right)=4.416$. Here, the excitation frequency interval has been discretized in 600 equidistant steps. The number of eigenmodes in the modal basis, at each iteration of the optimization algorithm, is defined such that the maximum considered eigenfrequency is at least twice the highest excitation frequency, i.e. $\max _{i} \omega_{i} \geq 2 \omega_{\max }$. More precisely, at each iteration, to determine the number of modes, we perform a loop, starting from the first ten eigenfrequencies and multiplying the number of modes by two, until the relation $\max _{i} \omega_{i} \geq 2 \omega_{\max }$ is satisfied. As a result of this choice, the number of 
considered eigenmodes can change during the optimization. For example, for the result of Figure 3 , the number of modes for the initial shape is 10 , while for the optimized shape it has increased to 40 .

The goal of this subsection is now to compare the value of the dynamic compliance $C_{d y n}\left(\Omega^{o p t}\right)$ for the shape $\Omega^{o p t}$ of Figure 3 when the number of modes in the modal basis and the number of discretized excitation eigenfrequencies vary. Table 1 reports the values of $C_{d y n}\left(\Omega^{o p t}\right)$ for different numbers of modes and frequencies. The differences are negligible, in particular when we choose $\max _{i} \omega_{i} \geq 2 \omega_{\max }$. Moreover, the dynamic compliance computed without the modal basis, namely solving the direct problem (5) and (6) for every excitation frequency (with 600 discretized frequencies), turns out to be equal to 4.418. It implies that the modal decomposition method converges to the standard approach when the number of modes increases, as far as the dynamic compliance is concerned. For the other numerical examples in this work, we shall consider sufficient the precision provided by discretizing the excitation frequency interval in 600 steps and using enough modes such that $\max \omega_{i} \geq 2 \omega_{\max }$.

Finally, we take the opportunity of this simple test case to monitor the evolution of the first 10 eigenfrequencies during the optimization history of (73) (which leads to the the optimized shape $\Omega^{o p t}$ of Figure 3 ). Figure 4 shows that some of the eigenfrequencies tend to approach, then separate again without crossing, while others clearly cross each other (e.g. the second with the third eigenvalue around iteration 5). Of course, it is rather difficult to validate the crossing by looking at the eigenvalue evolution and a mode-tracking approach should provide more reliable conclusions. Nevertheless, it is a clear indication that multiple eigenvalues may arise and thus leads to difficulties in their derivation (if the method of Section 6 were to be used).

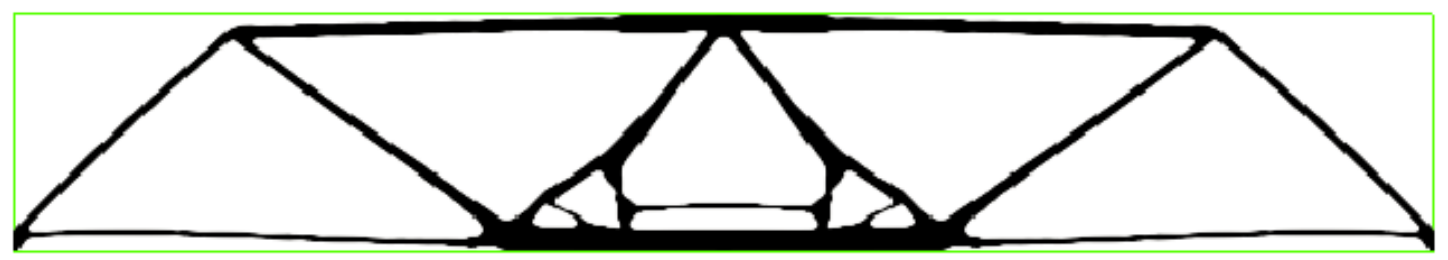

Figure 3: Optimized shape $\Omega^{o p t}$ for (73) with $\alpha=0.3$ and $C=2 \xi M$.

\begin{tabular}{|c|c|c|c|c|}
\hline discretization intervals & $\begin{array}{c}\max _{i} \omega_{i} \geq \omega_{\max } \\
(20 \text { eigenmodes })\end{array}$ & $\begin{array}{c}\max _{i} \omega_{i} \geq 2 \omega_{\max } \\
(40 \text { eigenmodes })\end{array}$ & $\begin{array}{c}\max _{i} \omega_{i} \geq 3 \omega_{\max } \\
(40 \text { eigenmodes })\end{array}$ & $\begin{array}{c}\max _{i} \omega_{i} \geq 4 \omega_{\max } \\
(80 \text { eigenmodes })\end{array}$ \\
\hline 100 & 4.383 & 4.417 & 4.417 & 4.419 \\
\hline 200 & 4.382 & 4.416 & 4.416 & 4.418 \\
\hline 300 & 4.382 & 4.416 & 4.416 & 4.418 \\
\hline 400 & 4.382 & 4.416 & 4.416 & 4.418 \\
\hline 500 & 4.382 & 4.416 & 4.416 & 4.418 \\
\hline 600 & 4.382 & 4.416 & 4.416 & 4.418 \\
\hline
\end{tabular}

Table 1: Dynamic compliance for the shape $\Omega^{\text {opt }}$ of Figure 3 for different number of eigenmodes in the modal basis and different discretizations of the excitation frequency interval $\left[\omega_{\min }, \omega_{\max }\right]$. 

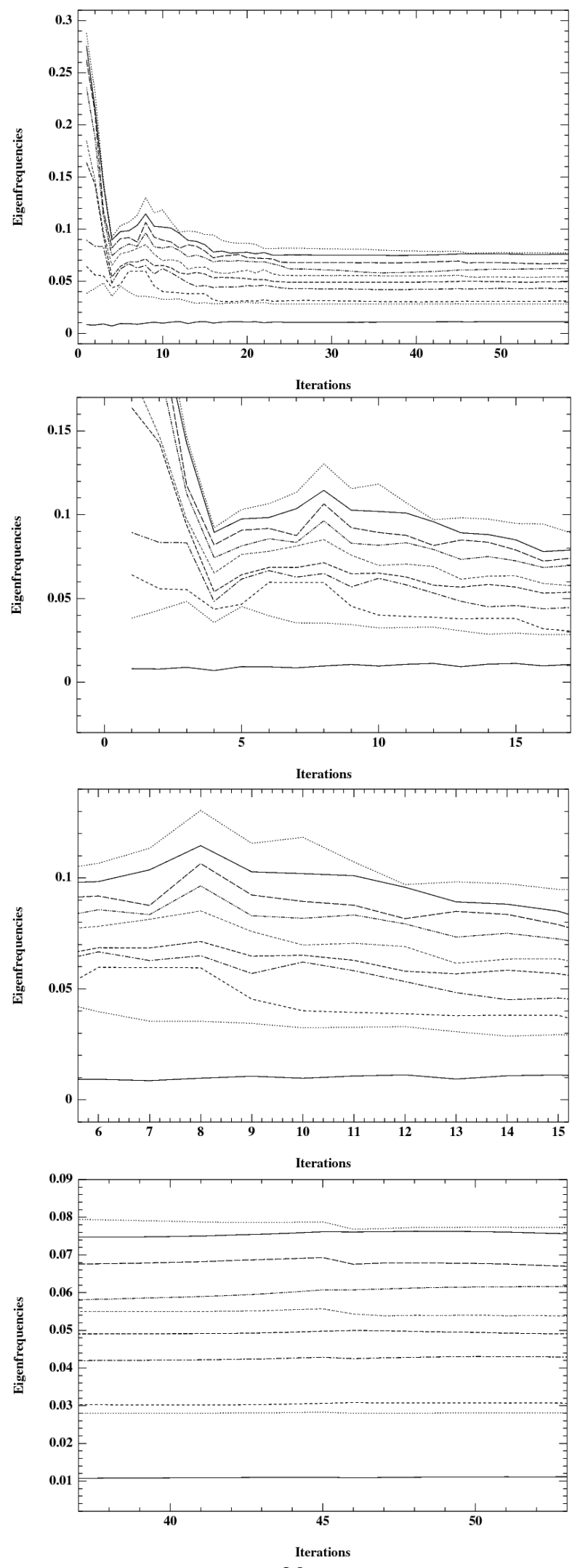

Figure 4: Evolution of the first 10 eigenfrequencies $\omega_{i}$ during the optimization process for the shape of Figure 3 (top) and various zooms (bottom). 
Remark 9.1. In this $M B B$ beam test case, we use a symmetry to reduce the size of the computational domain, and thus the CPU cost. Of course, this simplification eliminates all eigenmodes which are antisymmetric in the full domain and, furthermore, induces the symmetry of the solutions of the direct problem (5) and (6). The optimal structures may have been different if we did not use such a symmetry assumption.

Remark 9.2. Our algorithms are implemented in Scilab [7] for the ease of testing. However Scilab, being an interpreted language, is notably inefficient if all algorithmic loops are not vectorized. Unfortunately, the computations of the coefficients in the modal basis is quite involved and we did not succeed in vectorizing those computations. Therefore we are unable to give fair CPU time comparisons between the different approaches we have discussed. We leave this task for future work. Just to give an idea, the entire optimization process for the shape of Figure 3 requires of the order of 13 hours of CPU time.

\section{Numerical results}

We proceed now with numerical results using the three modal basis approaches presented before, i.e. the adjoint method of Section 5 , the modal decomposition of the eigenvectors' shape derivatives of Section 6 and the modal decomposition of the direct and adjoint states of Section 7. For the two-dimensional cases, we use Scilab [7], while in three dimensions we rely on FreeFem ++ [13] instead, so as to overcome problems related to the memory size [4]. For all examples here, a SLP-type optimization algorithm has been used, similar to the one presented in [11].

For all examples, the Young modulus $E$ and the density $\rho$ of the elastic material have been normalized to 1 , while for the "ersatz" material the values $E=10^{-3}$ and $\rho=10^{-5}$ have been chosen, so as to avoid the appearance of spurious modes, localized in the ersatz material. The Poisson ratio of both materials is set to 0.33 . The damping coefficients are modeled as $C_{k k}=$ $2 \xi \omega_{k} M_{k k}=2 \xi \omega_{k}$, with $\xi=0.05$.

Based on the results of Section 9, the excitation frequency interval is discretized using 600 equidistant steps to ensure sufficient accuracy, while for the modal basis, as explained previously, starting from the first ten eigenfrequencies we perform a loop, multiplying the total number by two at each step, until the relation $\max _{i} \omega_{i} \geq 2 \omega_{\max }$ is satisfied.

\subsection{Two-dimensional MBB beam}

Our first example is again the two-dimensional MBB beam of Section 9 (see Figure 1 for the boundary and loading conditions). We solve problem (73), which involves a bound on the dynamic compliance, for an external force of the type $\cos (\omega t)(0,1)$ in the frequency range $[0.02,0.1] \mathrm{Hz}$. The reference shape $\Omega^{\text {ref }}$ is again that of Figure 2 .

The optimized shapes for problem (73), using all three methods (of Sections 5, 6 and 7), for different values of the upper bound coefficient $\alpha$, appear in Figures 5, 6 and 7. The values of the objective function and constraints, as well as the number of optimization iterations, are shown in Table 2. From these results, one can easily verify that none of the three methods outperforms the others, both in terms of objective function value or total iteration numbers. Moreover, as expected, all results correspond to local minima, which depend strongly on the initialization. This becomes evident from the observation that the shape obtained using the modal decomposition of the eigenvectors' shape derivative for $\alpha=0.3$ has lower volume than the optimized shape without considering the dynamic compliance. As a result, modifying the optimization parameters could result in another local optimum. For example, compared to the optimized shape of Figure 6 (up), constraining the maximum number of transport iteration steps for the level-set function to the 
half results in another optimized shape, shown in Figure 8. The performance of this new shape is slightly better than previsouly $\left(V(\Omega)=0.431, C(\Omega)=148.19, C_{d y n}(\Omega)=10.21\right)$, however it requires more iterations to converge (46 iterations instead of 33 previously).
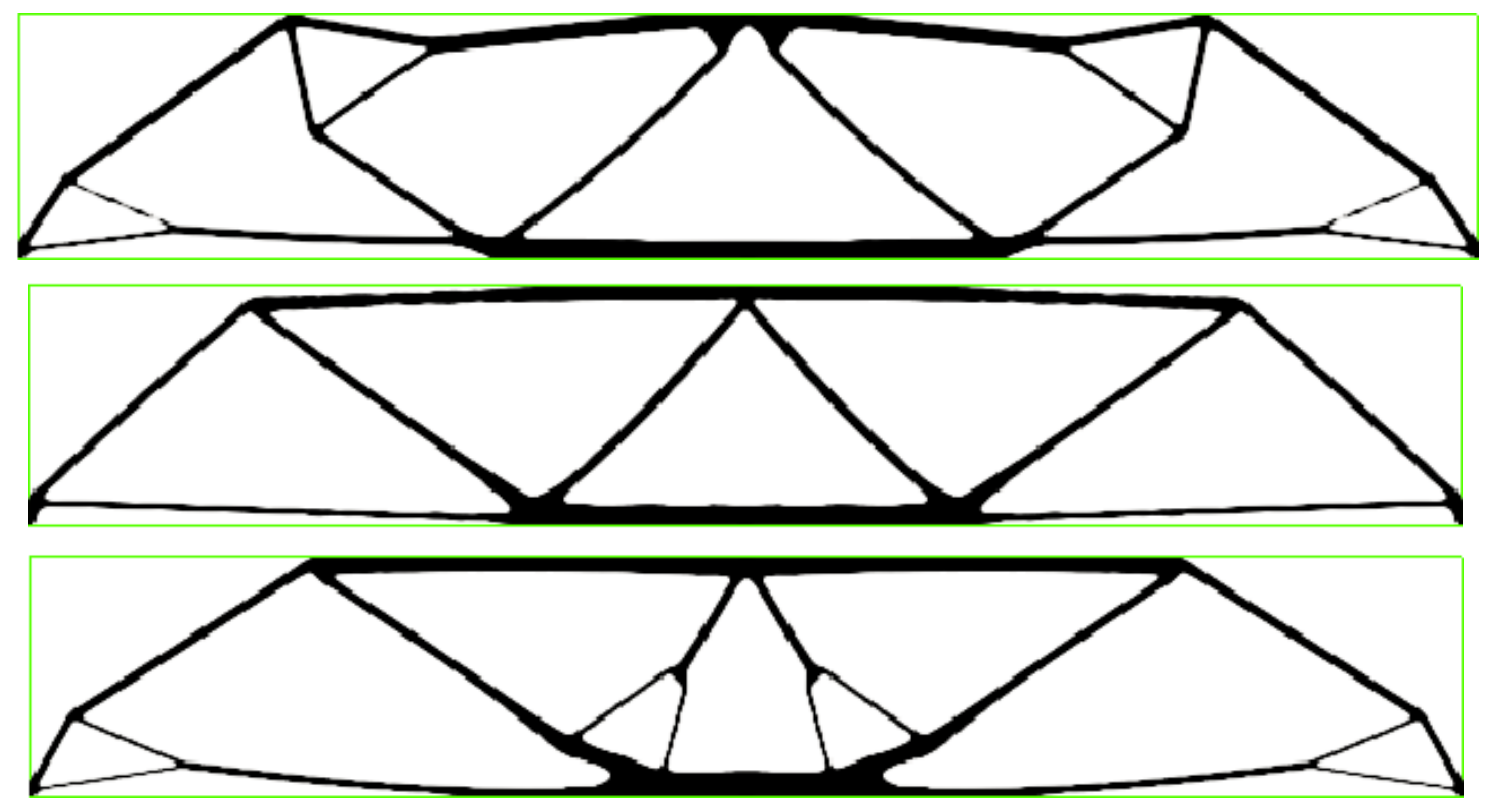

Figure 5: Optimized MBB beam for problem (73) setting: $\alpha=0.70$ (up); $\alpha=0.50$ (middle); $\alpha=0.30$ (down), using the adjoint method of Section 5 .
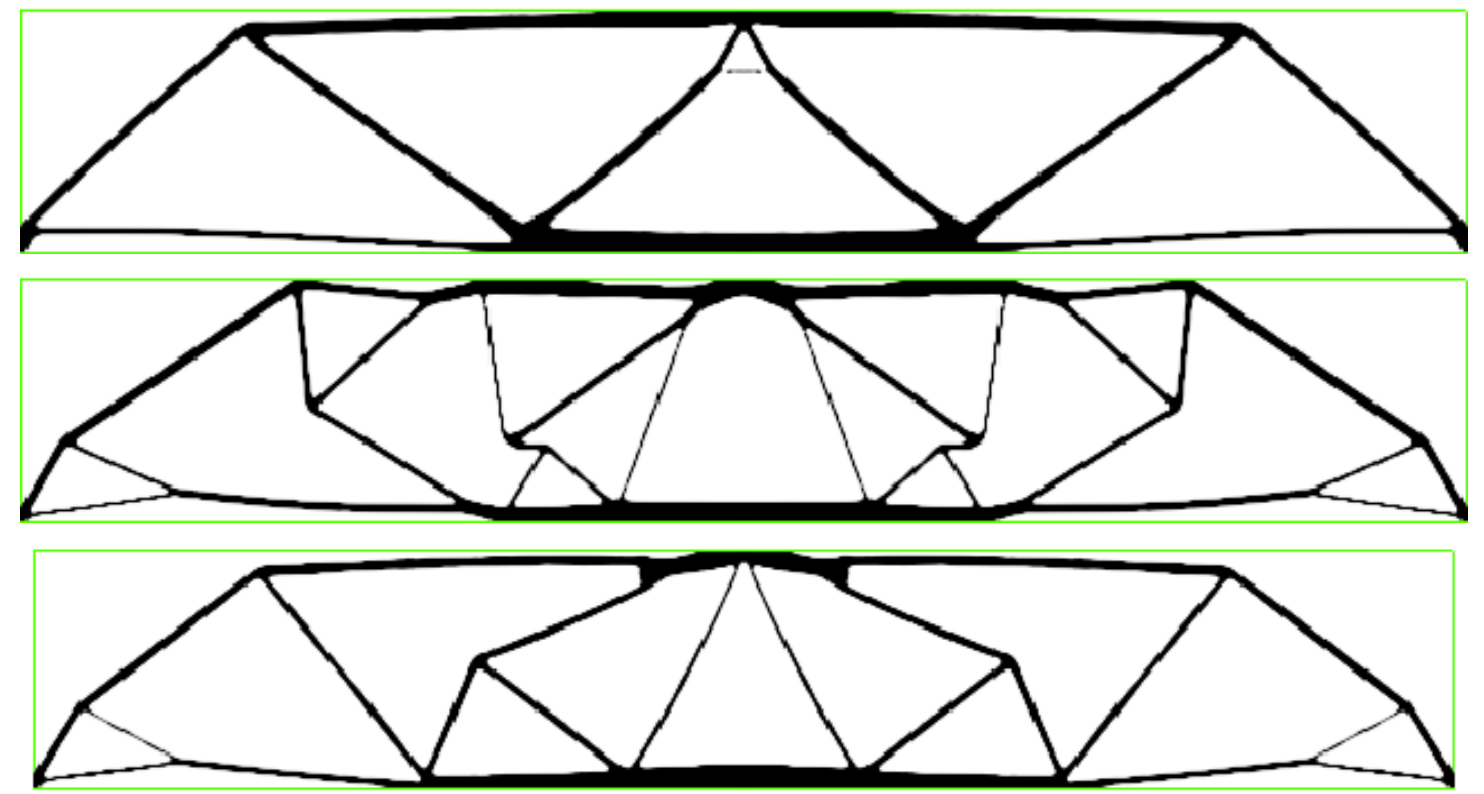

Figure 6: Optimized MBB beam for problem (73) setting: $\alpha=0.70$ (up); $\alpha=0.50$ (middle); $\alpha=0.30$ (down), using the modal decomposition of eigenvectors' shape derivative of Section 6 . 

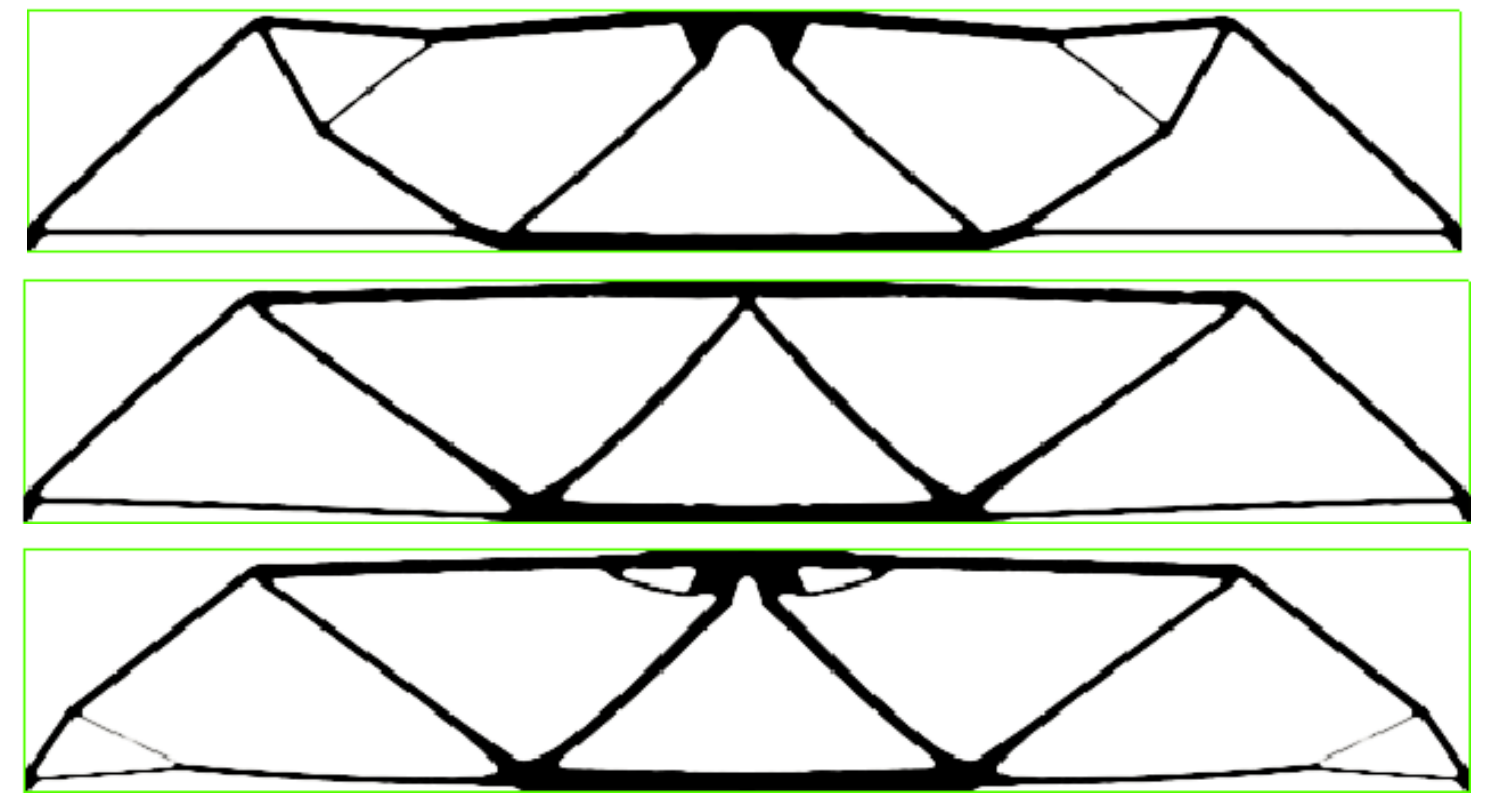

Figure 7: Optimized MBB beam for problem (73) setting: $\alpha=0.70$ (up); $\alpha=0.50$ (middle); $\alpha=0.30$ (down), using the modal decomposition of the direct and adjoint states of Section 7 .

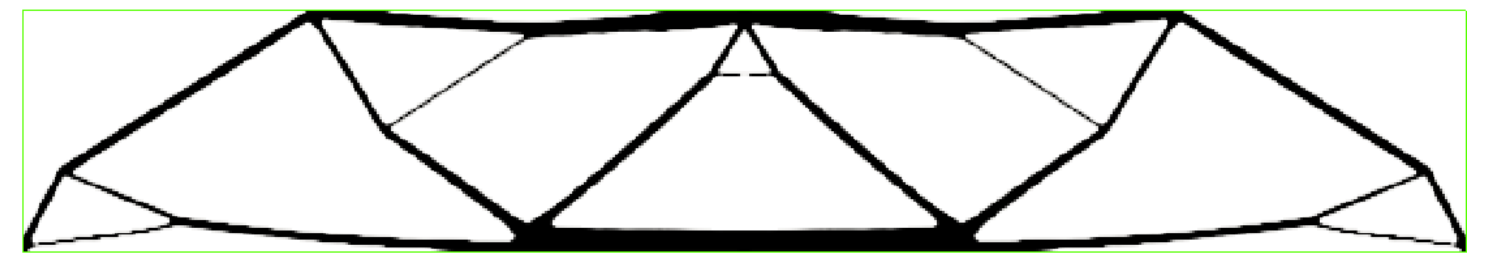

Figure 8: Optimized MBB beam for problem (73) setting: $\alpha=0.70$, using the modal decomposition of eigenvectors' shape derivative of Section 6 and constraining the advection transport steps to the half compared to Figure 6 (up).

\begin{tabular}{|c|c|c|c|c|c|c|}
\hline Method & $\alpha$ & $V(\Omega)$ & $C(\Omega)$ & $C_{d y n}(\Omega)$ & $\alpha C_{d y n}\left(\Omega^{\text {ref }}\right)$ & Iterations \\
\hline Problem (72), $C_{\max }=150$ & - & 0.415 & 149.99 & 14.83 & - & 22 \\
\hline & 0.70 & 0.431 & 149.78 & 10.18 & 10.38 & 23 \\
Adjoint approach & 0.50 & 0.427 & 149.10 & 7.33 & 7.42 & 35 \\
& 0.30 & 0.442 & 149.95 & 4.37 & 4.45 & 23 \\
\hline Modal decomposition & 0.70 & 0.434 & 149.27 & 10.03 & 10.38 & 33 \\
of eigenvectors' & 0.50 & 0.437 & 149.78 & 7.28 & 7.42 & 65 \\
shape derivative & 0.30 & 0.414 & 149.95 & 4.26 & 4.45 & 114 \\
\hline Modal decomposition & 0.70 & 0.430 & 149.37 & 10.22 & 10.38 & 29 \\
of direct and adjoint & 0.50 & 0.430 & 149.63 & 7.27 & 7.42 & 25 \\
states & 0.30 & 0.467 & 149.99 & 4.45 & 4.45 & 48 \\
\hline
\end{tabular}

Table 2: Results for the two-dimensional MBB beam. 


\subsection{Two-dimensional cantilever}

The second example is a two-dimensional 2x1 cantilever, discretized by 200x100 Q1 elements, clamped at its left boundary and with a unit vertical force applied at the middle of its right side (see Figure 9). The initialization and the optimized shape for problem (72) (without the dynamic compliance), choosing $C_{\max }=170$, appear in Figure 10. It will serve as the reference shape in the sequel.

Figures 11, 12 and 13 show the optimized shapes for problem (73) for all three methods and different values of $\alpha$. The vibrating force is of the same type as previously and the excitation frequency belongs to the interval $\omega \in[0.1,0.3] \mathrm{Hz}$. The reference value for the dynamic compliance equals $C_{d y n}\left(\Omega^{r e f}\right)=57.40$. More details about these results are presented in Table 3 .

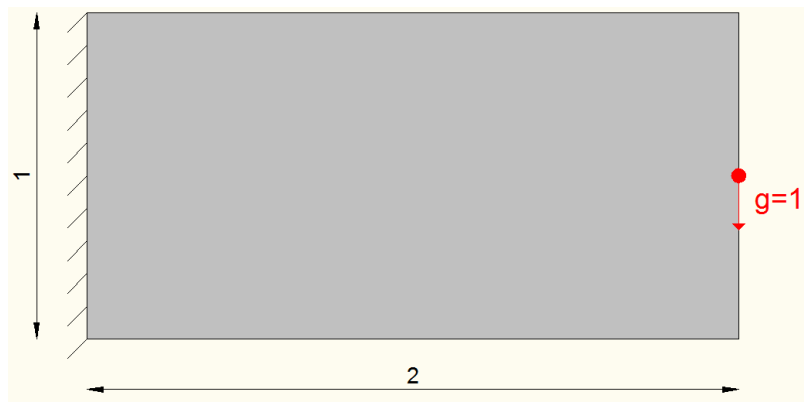

Figure 9: Boundary conditions for the two-dimensional cantilever.
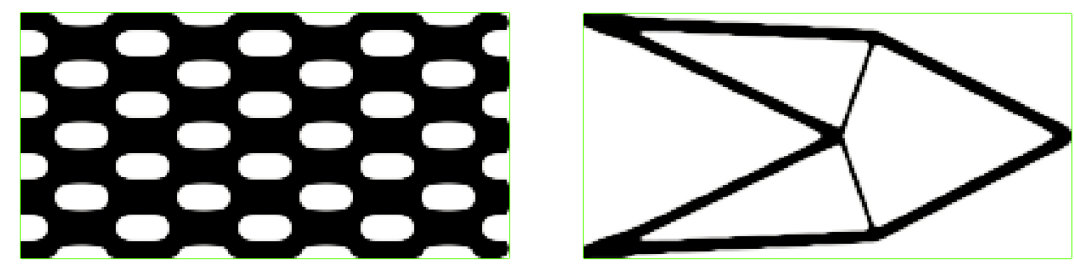

Figure 10: Left: initialization; right: optimized shape for the static compliance problem (72).
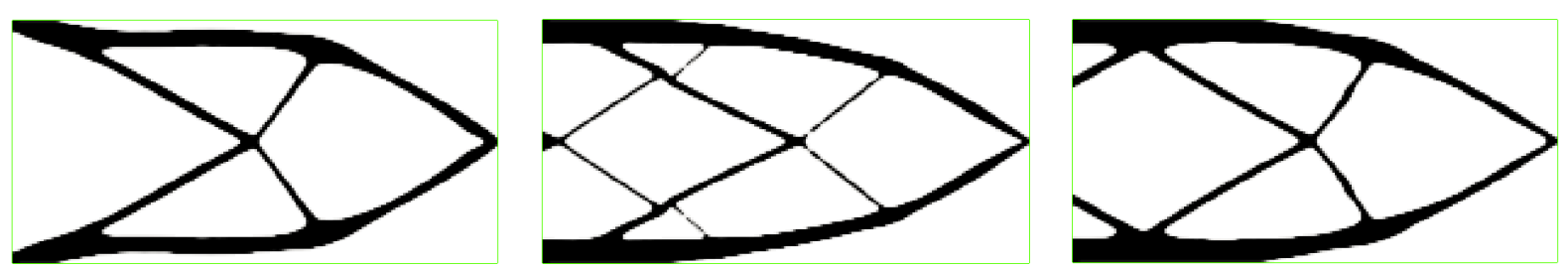

Figure 11: Optimized cantilever for problem (73) setting: $\alpha=0.70$ (left); $\alpha=0.50$ (middle); $\alpha=0.30$ (right), using the adjoint method of Section 5 . 

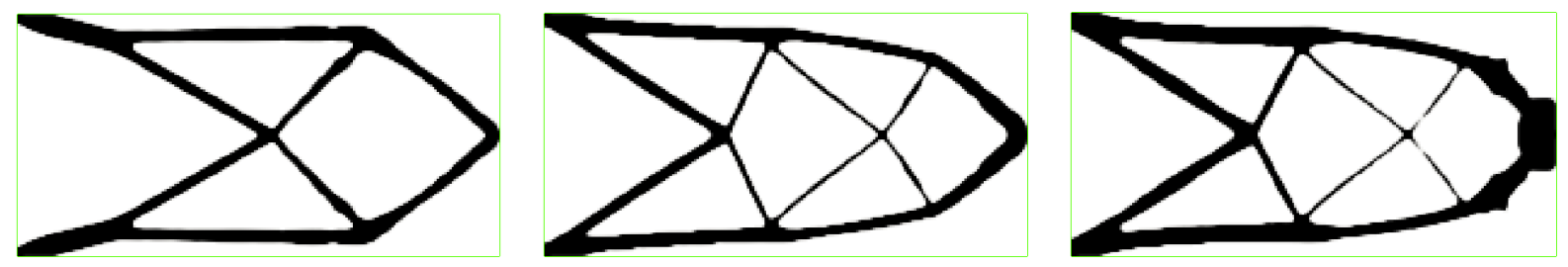

Figure 12: Optimized cantilever for problem (73) setting: $\alpha=0.70$ (left); $\alpha=0.50$ (middle); $\alpha=0.30$ (right), using the modal decomposition of eigenvectors' shape derivatives of Section 6 .
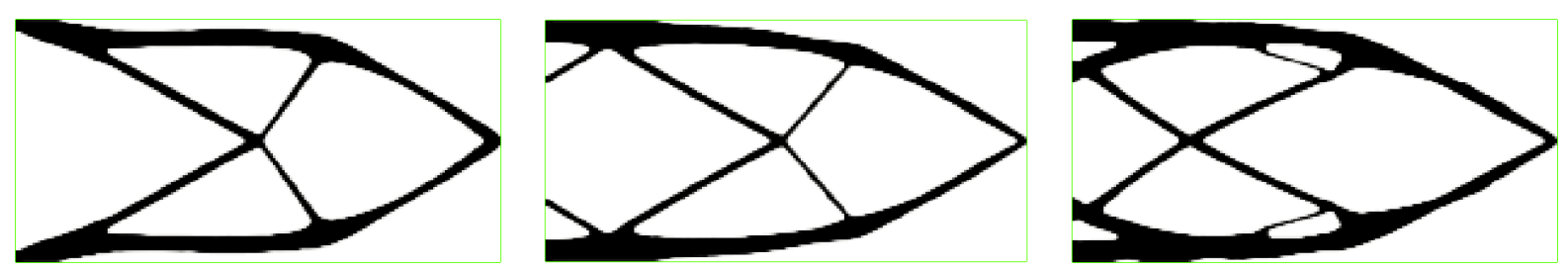

Figure 13: Optimized cantilever for problem (73) setting: $\alpha=0.70$ (left); $\alpha=0.50$ (middle); $\alpha=0.30$ (right), using the modal decomposition of the direct and adjoint states of Section 7 .

\begin{tabular}{|c|c|c|c|c|c|c|}
\hline Method & $\alpha$ & $V(\Omega)$ & $C(\Omega)$ & $C_{d y n}(\Omega)$ & $\alpha C_{d y n}\left(\Omega^{\text {ref }}\right)$ & Iterations \\
\hline Problem (72), $C_{\max }=170$ & - & 0.084 & 169.83 & 57.40 & - & 20 \\
\hline & 0.70 & 0.092 & 169.70 & 39.99 & 40.18 & 37 \\
Adjoint & 0.50 & 0.096 & 169.77 & 28.41 & 28.70 & 112 \\
& 0.30 & 0.103 & 169.99 & 17.22 & 17.22 & 115 \\
\hline Modal decomposition & 0.70 & 0.092 & 169.85 & 39.27 & 40.18 & 16 \\
of eigenvectors' & 0.50 & 0.094 & 169.92 & 27.71 & 28.70 & 22 \\
shape derivative & 0.30 & 0.115 & 168.94 & 15.43 & 17.22 & 35 \\
\hline Modal decomposition & 0.70 & 0.092 & 169.98 & 39.37 & 40.18 & 29 \\
of direct and adjoint & 0.50 & 0.095 & 169.43 & 28.60 & 28.70 & 55 \\
states & 0.30 & 0.120 & 169.53 & 16.70 & 17.22 & 70 \\
\hline
\end{tabular}

Table 3: Results for the two-dimensional cantilever.

\subsection{Three-dimensional bridge}

The first three-dimensional example is a $6 \mathrm{x} 1 \mathrm{x} 1$ bridge-like structure. The displacement is fixed at the two lower-right corners and the vertical displacement is fixed at the opposite corners. A uniform pressure load $q$ is applied at the top of the bridge such that $\int_{\Gamma_{N}} q d s=100$. The boundary conditions are shown in Figure 14. Due to symmetry, only one quarter of the structure is considered and discretized by a $60 \times 20 \times 10$ uniform hexaedral mesh. The solution of the elasticity and eigenvalue problems is performed in FreeFem ++ , by splitting each cubic element of the mesh into 6 tetrahedra [12].

The optimized shape for problem (72) and $C_{\max }=7510250$ is displayed on Figure 15. Then, multipying this pressure load $q$ by a vibrating amplitude $\cos (\omega t)$ in the frequency interval $\omega \in$ 
[0.03, 0.08] Hz, we solve problem (73) with a bound on the dynamic compliance with respect to the reference structure of Figure 15, for which $C_{d y n}\left(\Omega^{r e f}\right)=186358$. For this test case, we use only the method of Section 7 which is based on the modal decomposition of the direct and adjoint states. For different values of $\alpha$, the optimized shapes appear in Figures 16, 17 and 18. More details for these results are presented in Table 4.

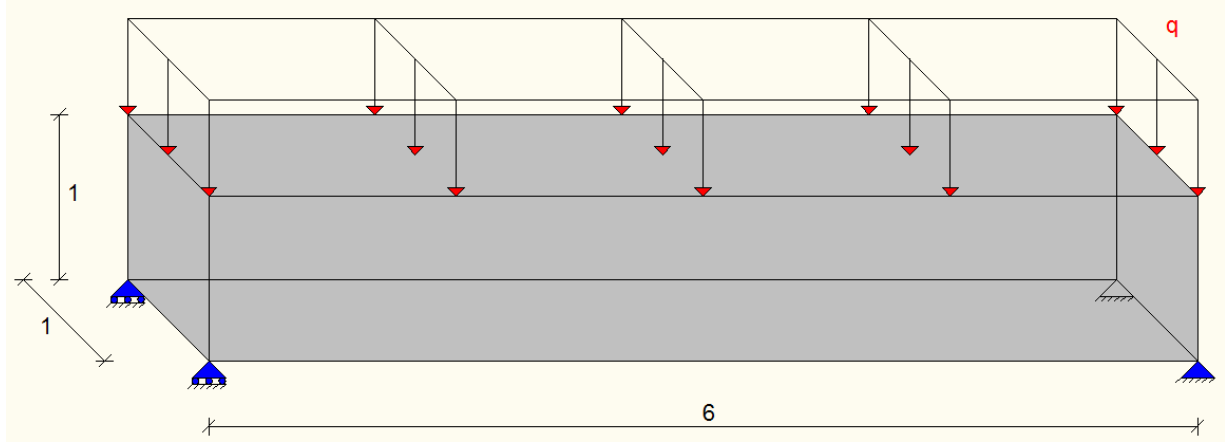

Figure 14: Boundary conditions for the three-dimensional bridge.
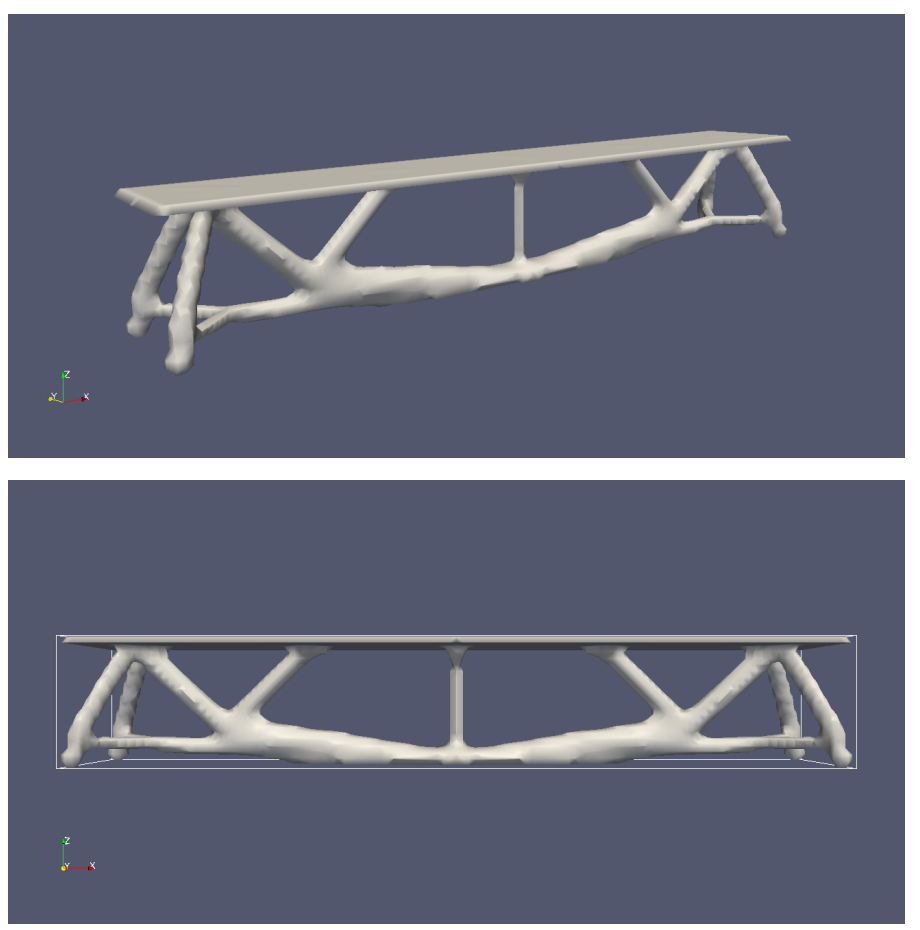

Figure 15: Optimized bridge for the static compliance problem (72). 

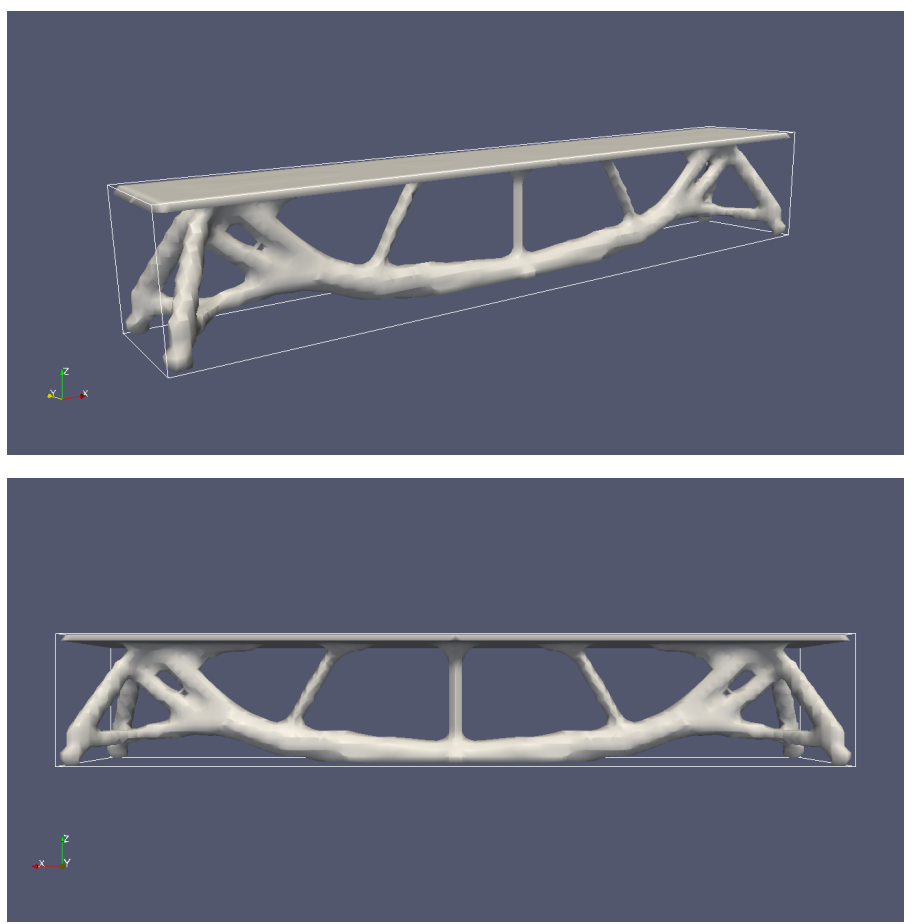

Figure 16: Optimized bridge for problem (73) setting $\alpha=0.70$, using the modal decomposition of the direct and adjoint states. 

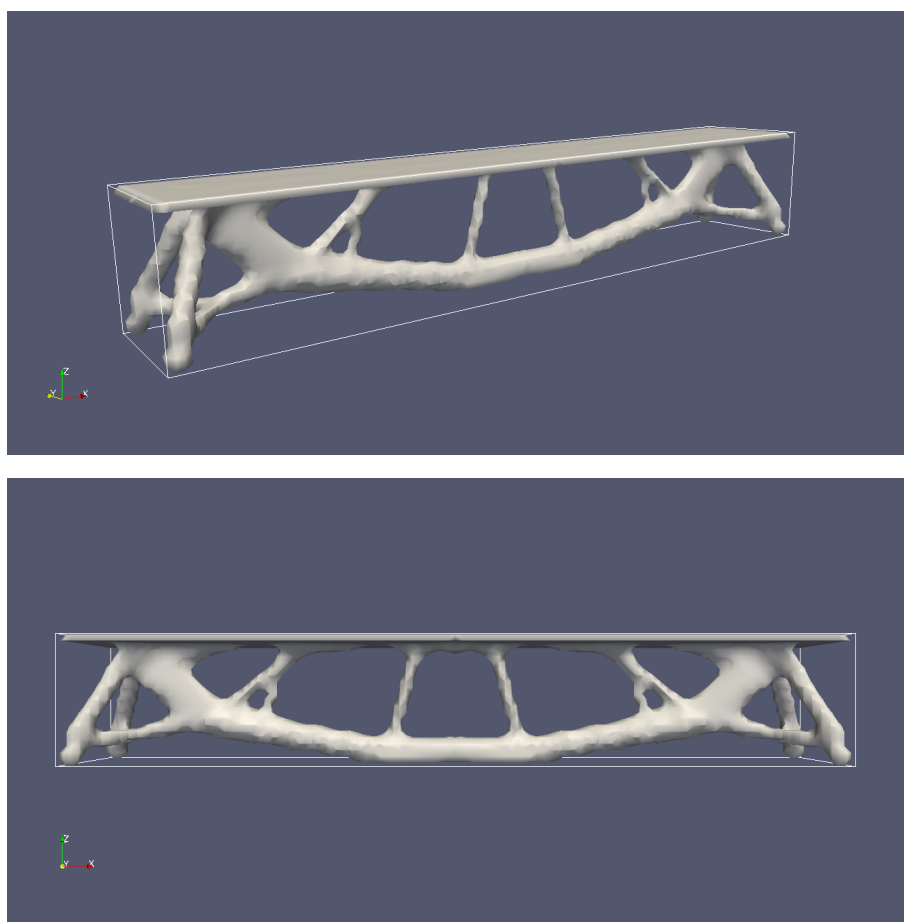

Figure 17: Optimized bridge for problem (73) setting $\alpha=0.50$, using the modal decomposition of the direct and adjoint states. 

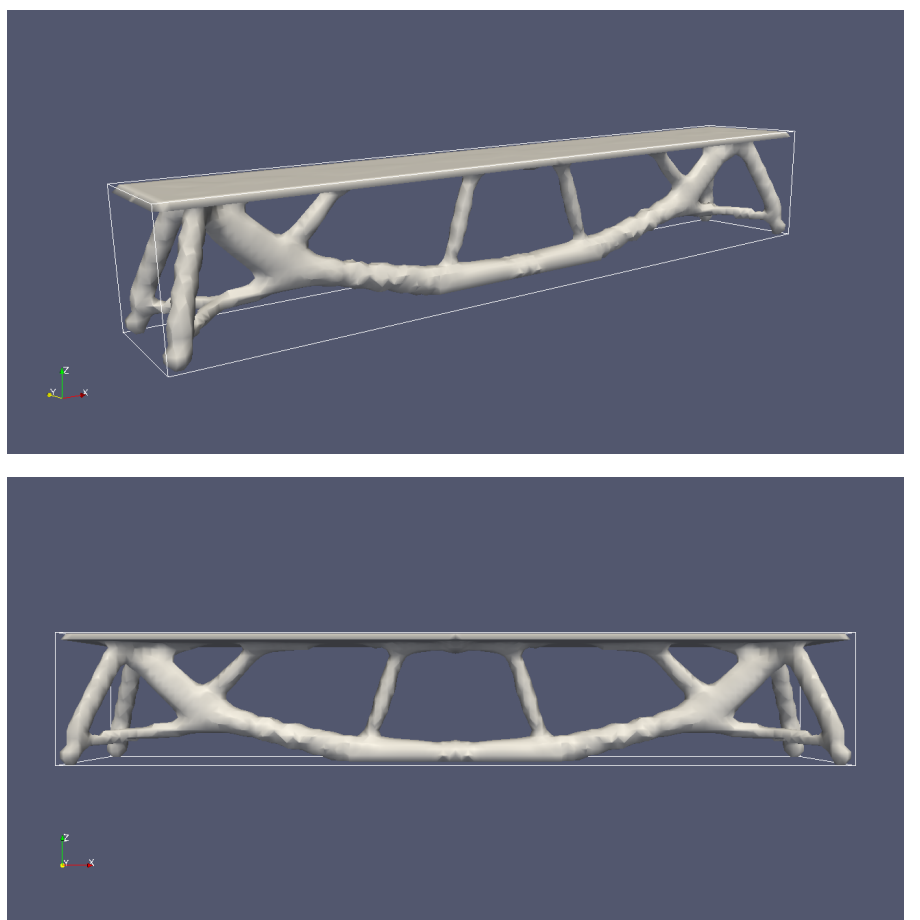

Figure 18: Optimized three-dimensional bridge for problem (73) setting $\alpha=0.30$, using the modal decomposition of the direct and adjoint states.

\begin{tabular}{|c|c|c|c|c|c|c|}
\hline Method & $\alpha$ & $V(\Omega)$ & $C(\Omega)$ & $C_{d y n}(\Omega)$ & $\alpha C_{d y n}\left(\Omega^{\text {ref }}\right)$ & Iterations \\
\hline Problem (72), $C_{\max }=7510250$ & - & 0.162 & 7479060 & 186358 & - & 49 \\
\hline Modal decomposition & 0.70 & 0.169 & 7506030 & 125195 & 130451 & 80 \\
of direct and adjoint & 0.50 & 0.174 & 7469840 & 91248 & 93179 & 44 \\
states & 0.30 & 0.167 & 7483880 & 51436 & 55907 & 58 \\
\hline
\end{tabular}

Table 4: Results for the three-dimensional bridge.

\subsection{Three-dimensional cantilever}

The last test-case is a three-dimensional cantilever, clamped at its left side and with a unit vertical load applied in the middle of its right side (see Figure 19). Due to symmetry with respect to the $y$-axis, only half of the domain is used and discretized by $60 \times 15 \times 30$ hexaedra.

The optimized shape for problem (72) and $C_{\max }=80$ is shown in Figure 20. Then, solving problem (73) for different values of $\alpha$, via the method of Section 7 (based on the modal decomposition of the direct and adjoint states), yields the optimized shapes of Figures 21, 22 and 23. The vibrating force is again the static load multiplied by $\cos (\omega t)$ in the excitation frequency interval $\omega \in[0.1,0.3] \mathrm{Hz}$. The value of the reference dynamic compliance is $C_{d y n}\left(\Omega^{r e f}\right)=18.41$. Table 5 contains the results for these shapes. 


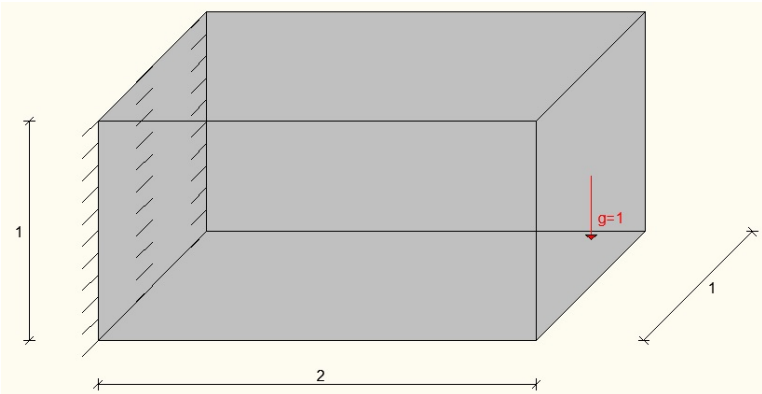

Figure 19: Boundary conditions for the three-dimensional cantilever.
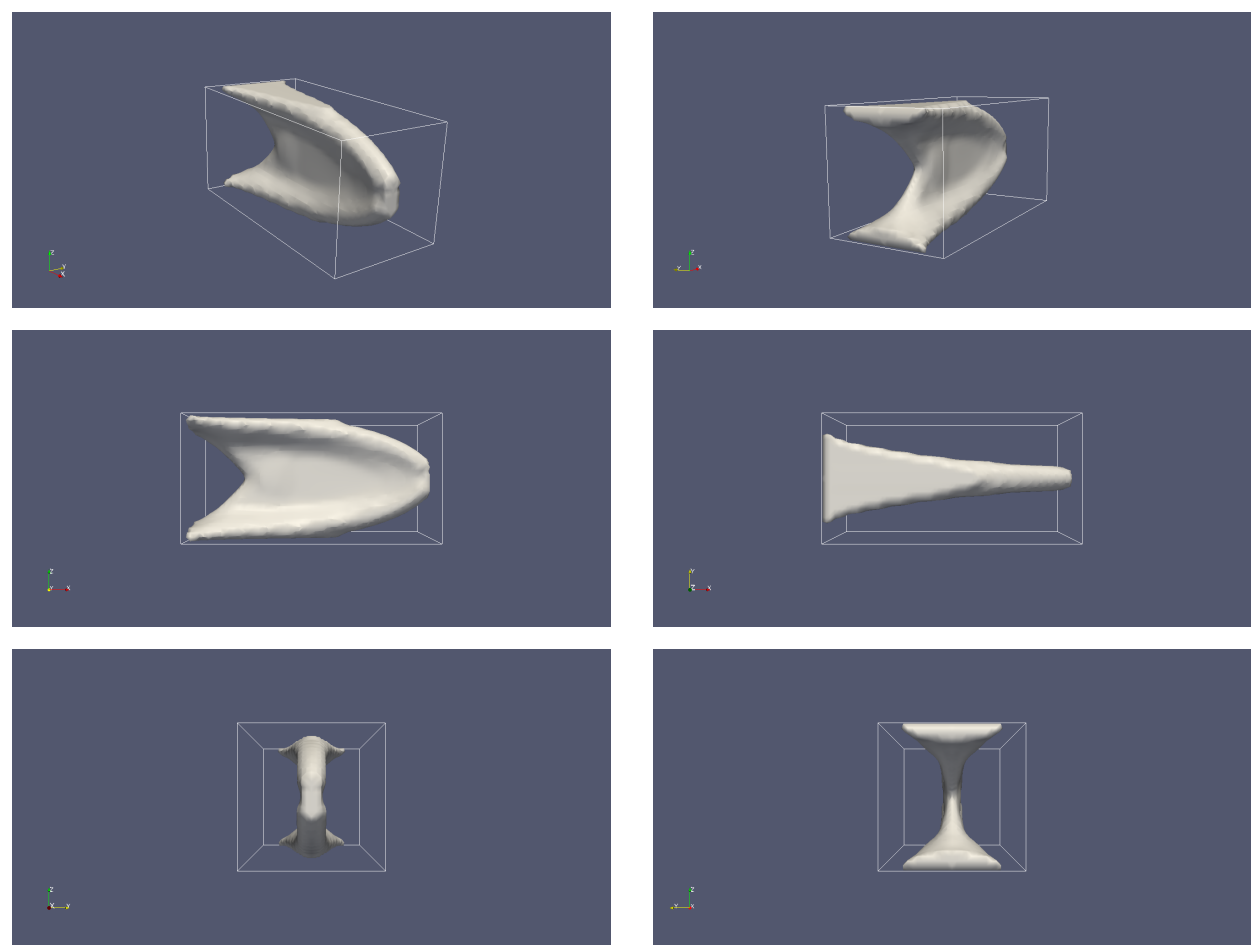

Figure 20: Optimized three-dimensional cantilever for problem (72). 

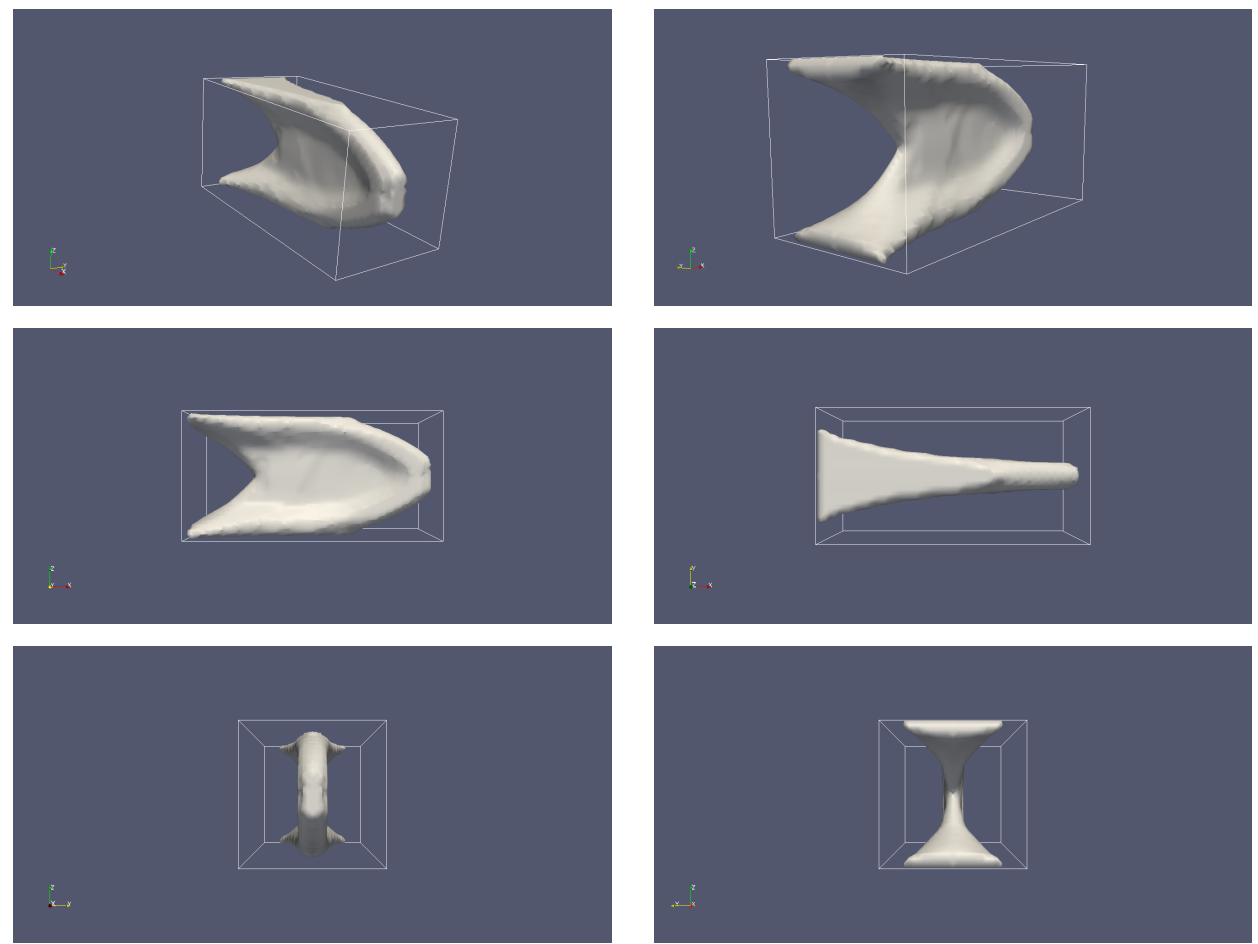

Figure 21: Optimized three-dimensional cantilever for problem (73) setting $\alpha=0.70$, using the modal decomposition of the direct and adjoint states. 

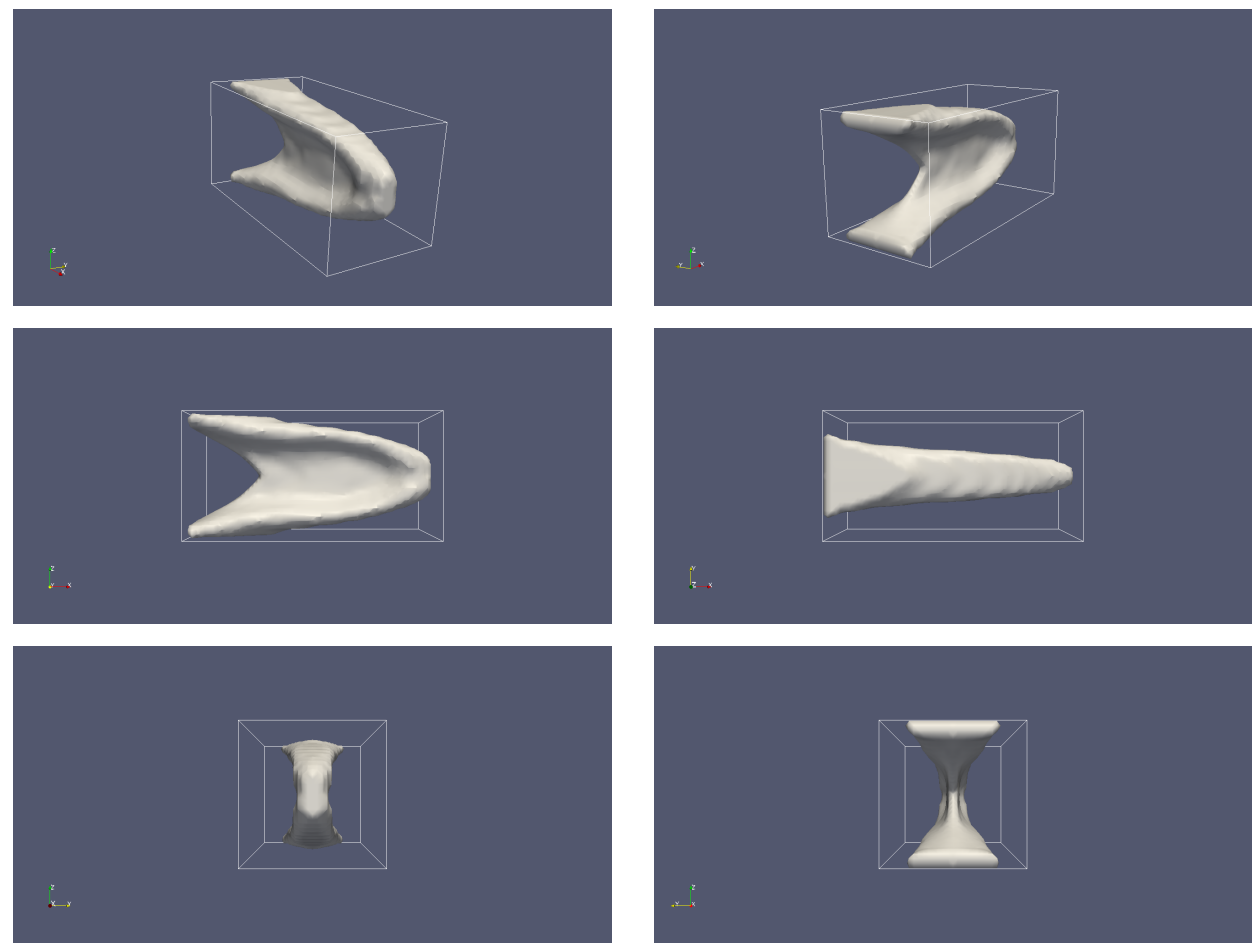

Figure 22: Optimized three-dimensional cantilever for problem (73) setting $\alpha=0.50$, using the modal decomposition of the direct and adjoint states. 

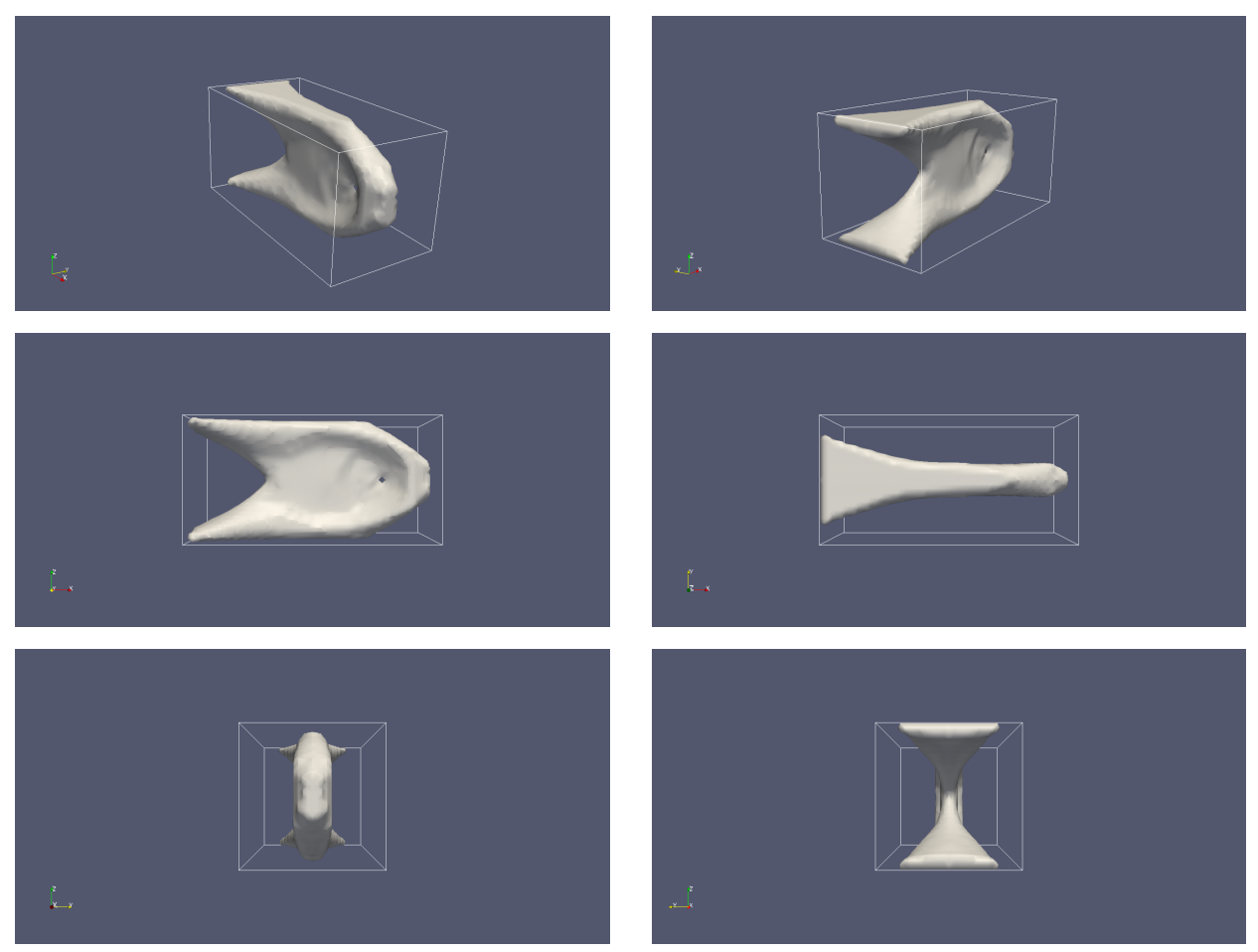

Figure 23: Optimized three-dimensional cantilever for problem (73) setting $\alpha=0.30$, using the modal decomposition of the direct and adjoint states.

\begin{tabular}{|c|c|c|c|c|c|c|}
\hline Method & $\alpha$ & $V(\Omega)$ & $C(\Omega)$ & $C_{d y n}(\Omega)$ & $\alpha C_{d y n}\left(\Omega^{\text {ref }}\right)$ & Iterations \\
\hline Problem (72), $C_{\max }=80$ & - & 0.162 & 79.94 & 18.41 & - & 49 \\
\hline Modal decomposition & 0.70 & 0.163 & 79.97 & 12.77 & 12.89 & 71 \\
of direct and adjoint & 0.50 & 0.175 & 79.96 & 9.16 & 9.21 & 28 \\
states & 0.30 & 0.167 & 79.95 & 5.38 & 5.52 & 81 \\
\hline
\end{tabular}

Table 5: Results for the three-dimensional cantilever.

\subsection{Double eigenvalues}

In order to test the non-sensitivity of the method with respect to the existence of multiple eigenvalues (see Remark (7.2)), we consider again the previous cantilever and we add a second load-case, similar to the first one, but with the external force oriented towards the $y$-axis. We discretize the full-domain via a $40 \times 20 \times 20$ hexaedral mesh and we do not use any symmetry assumption. Starting from a full-domain initialization and solving the optimization problem:

$$
\begin{array}{rl}
\min _{\Omega \in \mathcal{U}_{a d}} & V(\Omega) \\
\text { s.t. } & C^{1}(\Omega)=\int_{\Gamma_{N}} g_{1} \cdot u d s \leq C_{\max } \\
& C^{2}(\Omega)=\int_{\Gamma_{N}} g_{2} \cdot u d s \leq C_{\max }
\end{array}
$$


for $C_{\max }=250, g_{1}=[0,0,1]$ and $g_{2}=[0,1,0]$ results in the optimized shape of Figure 24. The optimized shape has kept its symmetry with respect to the $y$ and $z$-axis and thus contains at least one eigenvalue of multiplicity greater than one.

Adding a dynamic frequency constraint for each of the vibrating forces $g_{1} \cos (\omega t)$ and $g_{2} \cos (\omega t)$, in the excitation frequency interval $\omega \in[0.1,0.3] \mathrm{Hz}$, we solve the optimization problem:

$$
\begin{array}{rl}
\min _{\Omega \in \mathcal{U}_{a d}} & V(\Omega) \\
\text { s.t. } & C^{1}(\Omega)=\int_{\Gamma_{N}} g_{1} \cdot u d s \leq C_{\max } \\
& C^{2}(\Omega)=\int_{\Gamma_{N}} g_{2} \cdot u d s \leq C_{\max } \\
& C_{d y n}^{1}(\Omega) \leq \alpha C_{d y n}\left(\Omega^{\text {ref }}\right) \\
& C_{d y n}^{2}(\Omega) \leq \alpha C_{d y n}\left(\Omega^{\text {ref }}\right)
\end{array}
$$

for $\alpha=0.3$ and $C_{d y n}\left(\Omega^{r e f}\right)=51.17$. The optimized shape is shown in Figure 25 and the corresponding results in Table 6 . Finally, the convergence diagrams for the objective function and the constraints are shown in Figure 26 and the evolution of the first ten eigenfrequencies is plotted in Figure 27.

Clearly there are several double eigenvalues. The first and second eigenvalues, as well as the eighth and ninth remain double all along the optimization history. The fifth and sixth eigenvalues are double in the beginning of the optimization, but after the third iteration a crossing occurs and its the fourth and fifth eigenvalues that continue as double. However, the existence of multiple eigenvalues does not seem to cause any trouble in the convergence of the optimization as can be checked on the convergence history of Figure 26.
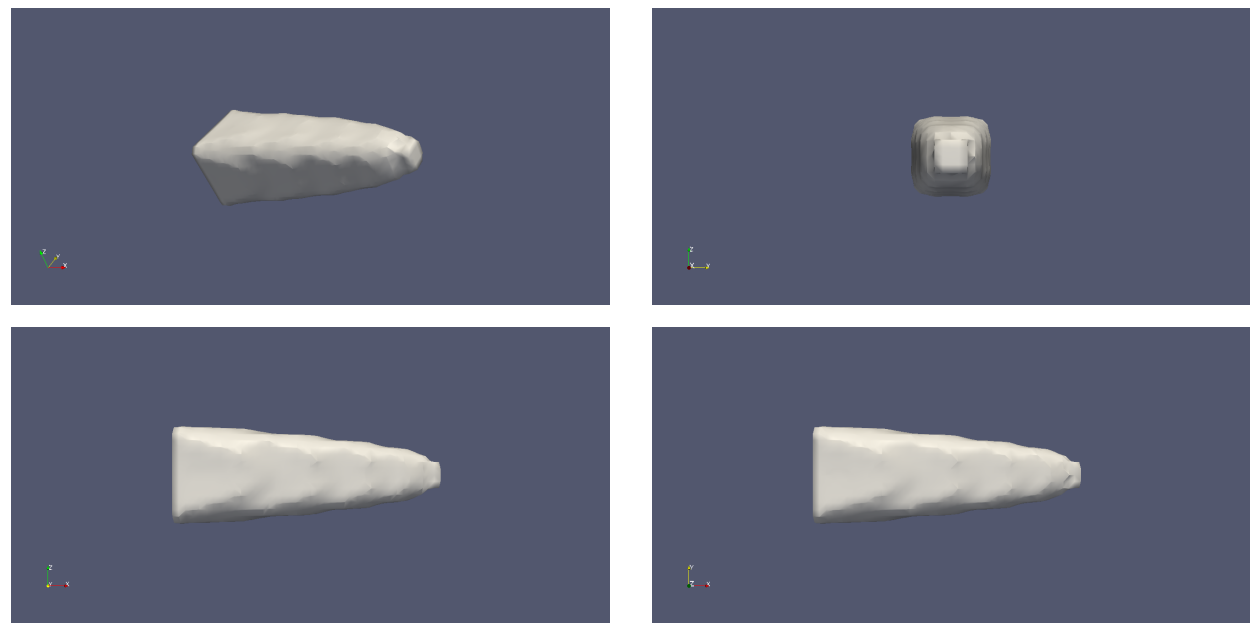

Figure 24: Optimized three-dimensional cantilever for problem (74). 

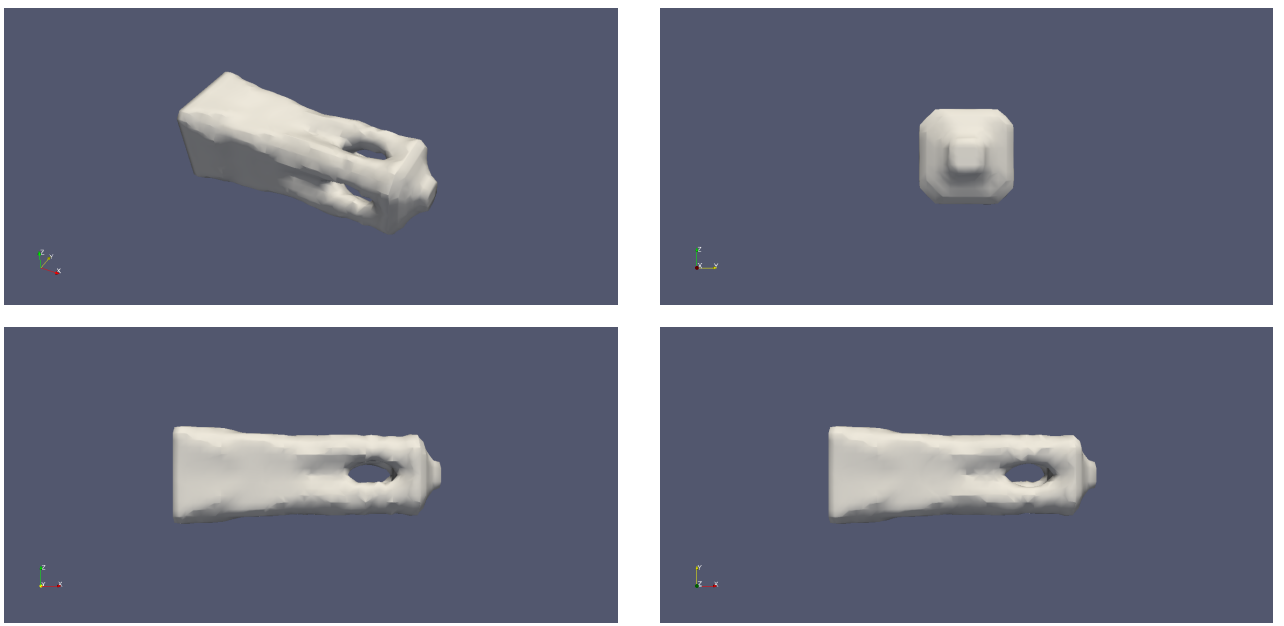

Figure 25: Optimized three-dimensional cantilever for problem (75) setting $\alpha=0.30$, using the modal decomposition of the direct and adjoint states.

\begin{tabular}{|c|c|c|c|c|c|c|c|c|}
\hline Method & $\alpha$ & $V(\Omega)$ & $C^{1}(\Omega)$ & $C^{2}(\Omega)$ & $C_{d y n}^{1}(\Omega)$ & $C_{d y n}^{2}(\Omega)$ & $\alpha C_{d y n}\left(\Omega^{\text {ref }}\right)$ & Iterations \\
\hline Problem (72), $C_{\max }=250$ & - & 0.620 & 249.493 & 249.493 & 51.17 & 51.17 & - & 13 \\
\hline $\begin{array}{c}\text { Modal decomposition } \\
\text { of direct and adjoint } \\
\text { states }\end{array}$ & 0.30 & 0.626 & 249.763 & 249.770 & 15.254 & 15.242 & 15.351 & 17 \\
\hline
\end{tabular}

Table 6: Results for the three-dimensional cantilever of figures 24 and 25.

\section{Acknowledgements}

The authors acknowledge fruitful discussions and helpful remarks from Marc Albertelli (Renault). The first author G.A. is a member of the DEFI project at INRIA Saclay Ile-de-France. This work started while the second author G.M. was a research engineer at CMAP, Ecole Polytechnique, financially supported by the Institut Carnot.

\section{References}

[1] G. Allaire. Conception optimale de structures, volume 58 of Mathématiques 8 Applications. Springer-Verlag, Berlin, 2007.

[2] G. Allaire, F. Jouve, and A.-M. Toader. A level-set method for shape optimization. C. R. Acad. Sci. Paris, Série I, 334:1125-1130, 2002.

[3] G. Allaire, F. Jouve, and A.-M. Toader. Structural optimization using sensitivity analysis and a level-set method. Journal of computational physics, 194(1):363-393, 2004.

[4] O. Amir, N. Aage, and B. Lazarov. On multigrid-cg for efficient topology optimization. Structural and Multidisciplinary Optimization, 49(5):815-829, 2014. 

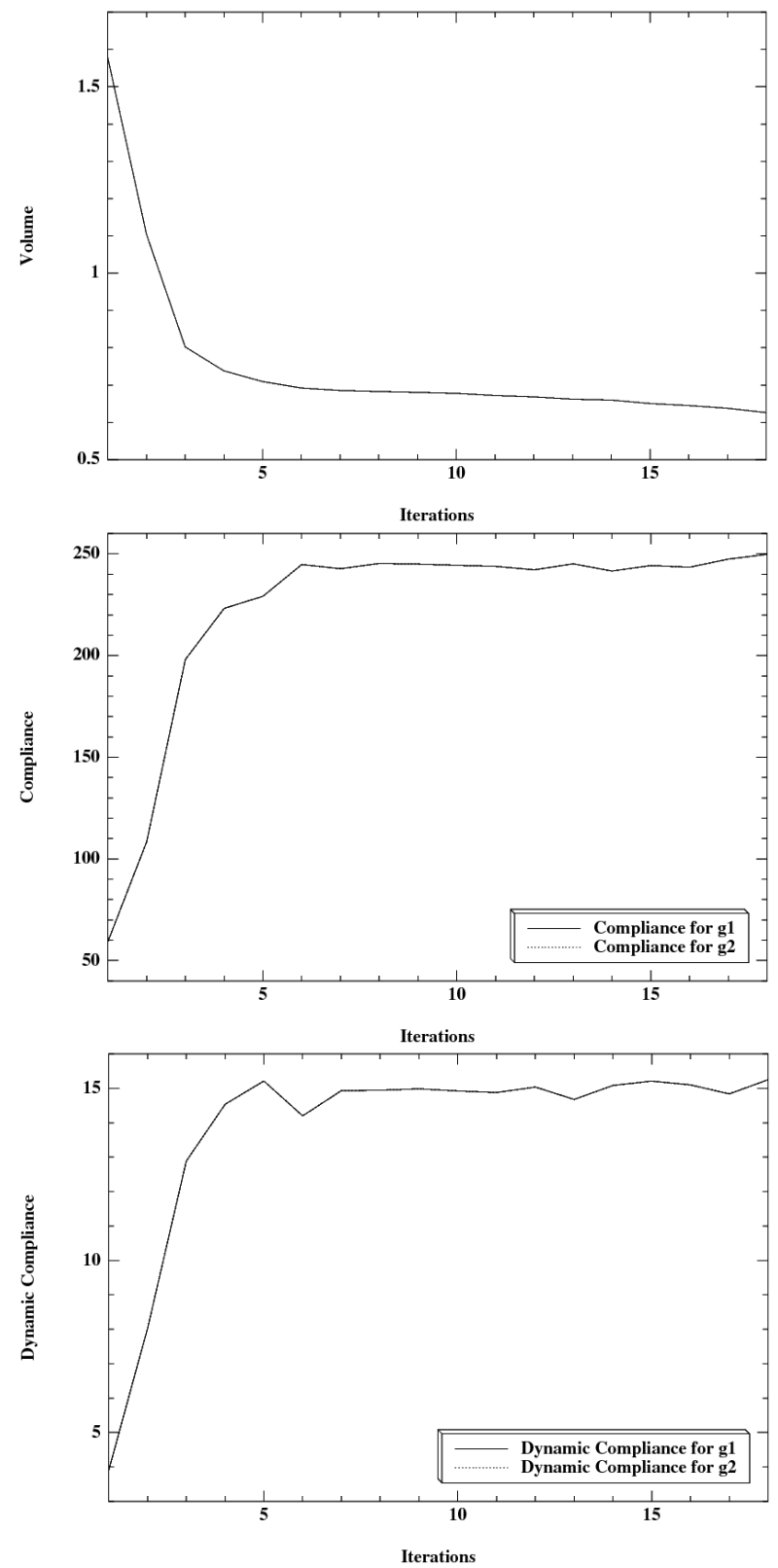

Figure 26: Convergence diagrams for the volume (up), the compliance (midle) and the dynamic compliance (down) for the results of Figure 25.

[5] M.P. Bendsoe and O. Sigmund. Topology optimization: theory, methods and applications. Springer, 2004.

[6] H. Brezis. Functional analysis, Sobolev spaces and partial differential equations. Universitext. Springer, New York, 2011.

[7] S. L. Campbell, J.-Ph. Chancelier, and R. Nikoukhah. Modeling and simulation in 
Scilab/Scicos. Springer, New York, 2006.

[8] J. Céa. Conception optimale ou identification de formes: calcul rapide de la dérivée directionnelle de la fonction coût. Modélisation mathématique et analyse numérique, 20(3):371-402, 1986.

[9] P.W. Christensen and A. Klarbring. An introduction to structural optimization, volume 153. Springer, 2009.

[10] R.W. Clough and J. Penzien. Dynamics of structures. McGraw-Hill Book Co., New York, 1975.

[11] P.D. Dunning and H.A. Kim. Introducing the sequential linear programming level-set method for topology optimization. Structural and Multidisciplinary Optimization, 51(3):631-643, 2015.

[12] P. Frey and H. Borouchaki. Texel: triangulation de surfaces implicites(partie ii, exemples d'applications). Rapports de recherche- INRIA, 1997.

[13] F. Hecht. New development in freefem++. J. Numer. Math., 20(3-4):251-265, 2012.

[14] A. Henrot and M. Pierre. Variation et optimisation de formes: une analyse géométrique, volume 48. Springer, 2005.

[15] J.S. Jensen. Topology optimization of dynamics problems with padé approximants. International Journal for Numerical Methods in Engineering, 72(13):1605-1630, 2007.

[16] C.S. Jog. Topology design of structures subjected to periodic loading. Journal of Sound and Vibration, 253(3):687-709, 2002.

[17] T. Kato. Perturbation theory for linear operators, volume 132 of Grundlehren der Mathematischen Wissenschaften. Springer Verlag, 1980.

[18] R. B. Lehoucq, D. C. Sorensen, and C. Yang. ARPACK users' guide, volume 6 of Software, Environments, and Tools. Society for Industrial and Applied Mathematics (SIAM), Philadelphia, PA, 1998.

[19] Z.-D. Ma, N. Kikuchi, and I. Hagiwara. Structural topology and shape optimization for a frequency response problem. Computational mechanics, 13(3):157-174, 1993.

[20] Zheng-Dong Ma, Noboru Kikuchi, and Hsien-Chie Cheng. Topological design for vibrating structures. Comput. Methods Appl. Mech. Engrg., 121(1-4):259-280, 1995.

[21] S. Min, N. Kikuchi, Y.C. Park, S. Kim, and S. Chang. Optimal topology design of structures under dynamic loads. Structural optimization, 17(2-3):208-218, 1999.

[22] Jean-Claude Nédélec. Acoustic and electromagnetic equations, volume 144 of Applied Mathematical Sciences. Springer-Verlag, New York, 2001. Integral representations for harmonic problems.

[23] N. Olhoff and J. Du. Topological design for minimum dynamic compliance of continuum structures subjected to forced vibration. Structural and Multidisciplinary Optimization, 2012.

[24] S.J. Osher and R. Fedkiw. Level set methods and dynamic implicit surfaces, volume 153 of Applied Mathematical Sciences. Springer-Verlag, New York, 2003. 
[25] S.J. Osher and F. Santosa. Level set methods for optimization problems involving geometry and constraints: I. frequencies of a two-density inhomogeneous drum. Journal of Computational Physics, 171(1):272-288, 2001.

[26] S.J. Osher and J.A. Sethian. Fronts propagating with curvature-dependent speed: algorithms based on hamilton-jacobi formulations. Journal of computational physics, 79(1):12-49, 1988.

[27] J.A. Sethian. Level set methods and fast marching methods: evolving interfaces in computational geometry, fluid mechanics, computer vision, and materials science. Cambridge university press, 1999.

[28] J.A. Sethian and A. Wiegmann. Structural boundary design via level set and immersed interface methods. Journal of computational physics, 163(2):489-528, 2000.

[29] L. Shu, M.Y. Wang, Z. Fang, Z. Ma, and P. Wei. Level set based structural topology optimization for minimizing frequency response. Journal of Sound and Vibration, 330(24):5820-5834, 2011.

[30] J. Simon and F. Murat. Sur le contrôle par un domaine géométrique. Publication 76015 du Laboratoire d'Analyse Numérique de l'Université Paris VI, (76015):222 pages, 1976.

[31] J. Sokołowski and J.-P. Zolésio. Introduction to shape optimization. Springer-Verlag, Berlin, 1992.

[32] D. Tcherniak. Topology optimization of resonating structures using simp method. International Journal for Numerical Methods in Engineering, 54(11):1605-1622, 2002.

[33] M.Y. Wang, X. Wang, and D. Guo. A level set method for structural topology optimization. Computer methods in applied mechanics and engineering, 192(1):227-246, 2003.

[34] G.H. Yoon. Structural topology optimization for frequency response problem using model reduction schemes. Computer Methods in Applied Mechanics and Engineering, 199(25):1744$1763,2010$. 

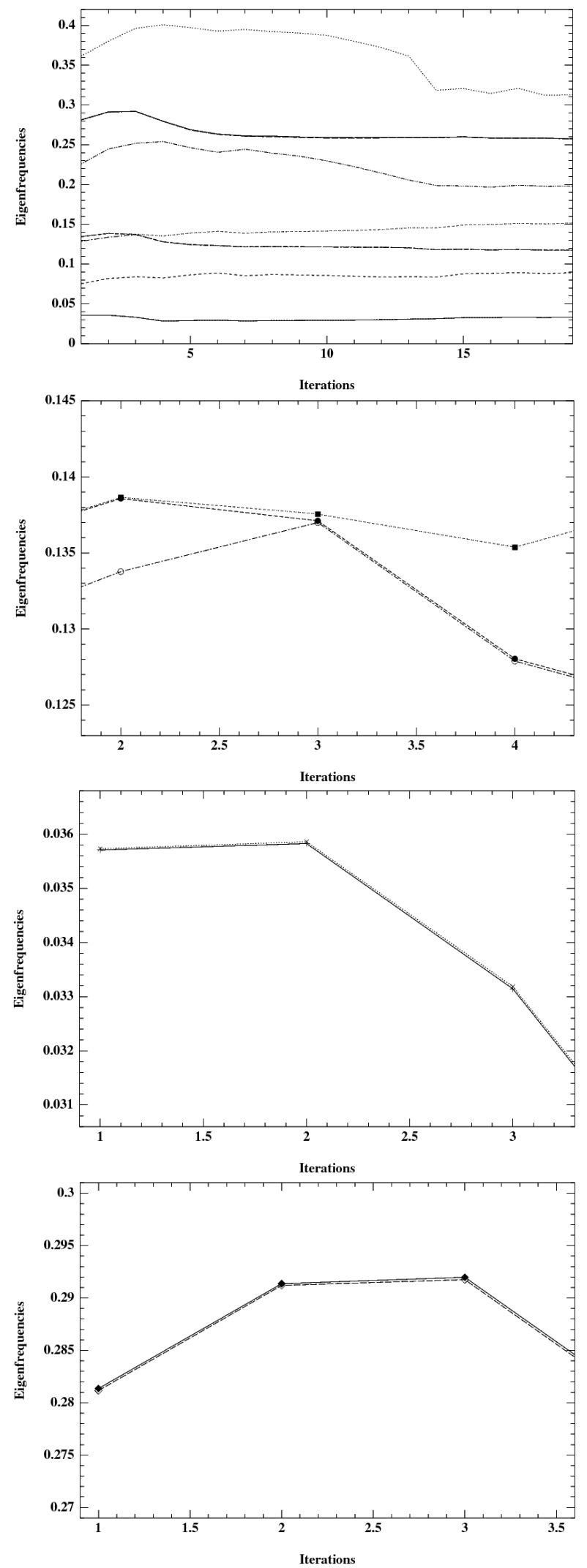

Figure 27: Evolution of the first 10 eigenfrequencies during the optimization process for the optimized shape of Figure 25 (top) and various zooms (bottom). 